\title{
Heat Entrapment Effects Within Liquid Acquisition Devices
}

W.M.B. Duval, D.J. Chato, and M.P. Doherty

Glenn Research Center, Cleveland, Ohio 


\section{NASA STI Program . . . in Profile}

Since its founding, NASA has been dedicated to the advancement of aeronautics and space science. The NASA Scientific and Technical Information (STI) program plays a key part in helping NASA maintain this important role.

The NASA STI Program operates under the auspices of the Agency Chief Information Officer. It collects, organizes, provides for archiving, and disseminates NASA's STI. The NASA STI program provides access to the NASA Aeronautics and Space Database and its public interface, the NASA Technical Reports Server, thus providing one of the largest collections of aeronautical and space science STI in the world. Results are published in both non-NASA channels and by NASA in the NASA STI Report Series, which includes the following report types:

- TECHNICAL PUBLICATION. Reports of completed research or a major significant phase of research that present the results of NASA programs and include extensive data or theoretical analysis. Includes compilations of significant scientific and technical data and information deemed to be of continuing reference value. NASA counterpart of peer-reviewed formal professional papers but has less stringent limitations on manuscript length and extent of graphic presentations.

- TECHNICAL MEMORANDUM. Scientific and technical findings that are preliminary or of specialized interest, e.g., quick release reports, working papers, and bibliographies that contain minimal annotation. Does not contain extensive analysis.

- CONTRACTOR REPORT. Scientific and technical findings by NASA-sponsored contractors and grantees.
- CONFERENCE PUBLICATION. Collected papers from scientific and technical conferences, symposia, seminars, or other meetings sponsored or cosponsored by NASA.

- SPECIAL PUBLICATION. Scientific, technical, or historical information from NASA programs, projects, and missions, often concerned with subjects having substantial public interest.

- TECHNICAL TRANSLATION. Englishlanguage translations of foreign scientific and technical material pertinent to NASA's mission.

Specialized services also include creating custom thesauri, building customized databases, organizing and publishing research results.

For more information about the NASA STI program, see the following:

- Access the NASA STI program home page at http://www.sti.nasa.gov

- E-mail your question via the Internet to help@ sti.nasa.gov

- Fax your question to the NASA STI Help Desk at 443-757-5803

- Telephone the NASA STI Help Desk at 443-757-5802

- Write to: NASA Center for AeroSpace Information (CASI) 7115 Standard Drive Hanover, MD 21076-1320 


\section{Heat Entrapment Effects Within Liquid Acquisition Devices}

W.M.B. Duval, D.J. Chato, and M.P. Doherty

Glenn Research Center, Cleveland, Ohio

Prepared for the

48th Aerospace Sciences Meeting

sponsored by the American Institute of Aeronautics and Astronautics Orlando, Florida, January 4-7, 2010

National Aeronautics and

Space Administration

Glenn Research Center

Cleveland, Ohio 44135 


\section{Acknowledgments}

This work is performed in support of the Cryogenic Fluid Management (CFM) program at NASA Glenn Research Center.

\section{Document Change History}

NASA/TM-2010-216789/REV1, July 2011

Heat Entrapment Effects Within Liquid Acquisition Devices

W.M.B. Duval, D.J. Chato, and M.P. Doherty

This printing replaces NASA/TM-2010-216789, September 2010, because extensive changes were made to the document.

Level of Review: This material has been technically reviewed by technical management.

Available from

NASA Center for Aerospace Information 7115 Standard Drive

Hanover, MD 21076-1320
National Technical Information Service 5301 Shawnee Road Alexandria, VA 22312

Available electronically at http://www.sti.nasa.gov 


\title{
Heat Entrapment Effects Within Liquid Acquisition Devices
}

\author{
W.M.B. Duval, D.J. Chato, and M.P. Doherty \\ National Aeronautics and Space Administration \\ Glenn Research Center \\ Cleveland, Ohio 44135
}

\begin{abstract}
We introduce a model problem to address heat entrapment effects or the local accumulation of thermal energy within liquid acquisition devices. We show that the parametric space consists of six parameters, namely the Rayleigh and Prandtl numbers, the aspect ratio, and heat flux ratios for the bottom, side, and top boundaries of the enclosure. For the range of Ra considered 1 to $10^{9}$, beyond Ra on the order of $10^{5}$, convective instability is the dominant mode of convection in comparison to natural convection. The flow field transitions to asymmetric modes at Ra on the order of $10^{7}$. Direct numerical simulation of a large geometric length scale prototype for Ra on the order of $10^{9}$ shows that the flow field evolves from small wavelength instability which gives rise to nonlinear growth of thermals, propagation of the instability occurs via growth of secondary and tertiary modes, and a travelling wave-type motion of convective modes occur prior to asymmetry. The effect of a large aspect ratio is to increase the number of modes in the vertical direction. Due to the slow diffusion of heat in the prototype, asymptotic states are not readily attained, we show that dynamical similarity can be used for a model which allows the attainment of asymptotic states and that transition to a chaotic state occurs for Ra on the order of $10^{9}$ via a broadband power spectrum. These dynamical events show that for the baseline condition in which heat is absorbed from background laboratory environment, higher heat flux is absorbed at the top and bottom boundaries of the enclosure than a nominal value of $34.9 \mathrm{erg} / \mathrm{cm}^{2}-\mathrm{s}$.
\end{abstract}

\section{Nomenclature}

$\begin{array}{ll}R a & \text { Rayleigh number } \\ A r & \text { aspect ratio } \\ \operatorname{Pr} & \text { Prandtl number } \\ f_{s} & \text { sidewall heat flux ratio } \\ f_{b} & \text { bottom heat flux ratio } \\ f_{t} & \text { top heat flux ratio } \\ q " & \text { imposed heat flux } \\ \mathrm{H} & \text { cavity height } \\ \mathrm{L} & \text { cavity width } \\ g & \text { gravitational acceleration } \\ k & \text { thermal conductivity } \\ \alpha & \text { thermal diffusivity } \\ \beta & \text { thermal expansion coefficient } \\ \rho & \text { density } \\ \Gamma & \text { boundary of cavity }\end{array}$




\section{Introduction}

Capillary liquid acquisition devices are used inside cryogenic storage tanks in order to supply vaporfree propellant to the engine. The liquid acquisition devise (LAD) interfaces directly with the feed system, for the supply of propellant, which can serve as a significant source of heat leak. In order to avoid excessive boil-off and ensure sufficient propellant subcooling to prevent cavitation and vapor formation during propellant outflow to the engine, the control of heat leak is necessary. Hence the localized accumulation of thermal energy, defined as heat entrapment, within the LAD flow channels becomes an issue of prime importance. We consider a model problem that addresses the effect of heat source at the boundary of an enclosure which contains typical cryogenic liquids (nitrogen, hydrogen) to lead insight into heat entrapment effects. This model problem has relevance towards the experimental works of Bolshinskiy et al. (Ref. 1), who considers heat entrapment effects modeled as controlled bottom heating of a cylindrical enclosure with and without partitions totally filled with a fluid. The partitions, which consist of typical screen meshes used in LADs as well as a solid divider, serve to emulate typical experimental conditions encountered during operations. Their results (Ref. 1) show that for the experimental conditions with partitions, the capillary screen meshes were impervious to natural convection. This is verified experimentally (Ref. 1) from comparison between data for meshes versus a solid boundary which show that the temperature rise are not significantly different. Hence a solid boundary can be used as an approximation of the boundary condition to address heat entrapment effects; this implies that the impermeable zero mass flux condition of the solid boundary models the boundary condition for the screen meshes. In our model we employ a solid boundary condition to address convection effects encountered in LAD applications.

The basic physics of heat entrapment effects or the localized accumulation of thermal energy is addressed by broadly considering the effect of heat flux absorption on the boundary of an enclosure, due to controlled heat flux on the bottom and unknown heat leakage at the top and side boundaries, of an enclosed cryogenic liquid initially at saturation condition which totally fills the volume of the enclosure. Of basic interest is the rise in temperature due to various external heat flux conditions. The quantification of the temperature field can be used to assess optimum operating conditions for propellant flow to the engine. Related to the broad problem of the effect of heat absorption on the temperature rise of a cryogenic fluid are also issues on effect of thermal stratification on temperature rise due to side heating of partially filled cavities with a free surface as addressed by Tellep and Harper (Ref. 2), Vliet et al. (Ref. 3), and Vliet (Ref. 4) with applications to cryogenic storage tanks. Approximate boundary layer analysis using an integral approach coupled with experiments (Refs. 2 and 3) show that the effect of dominant side heating is to transport warmer fluid along the wall to the interface region which becomes accumulated at the free interface thus forming a stratified layer. Vliet (Ref. 4) addressed the effect of bottom heating using an approximate integral approach and show agreement with experimental data; the results show that as bottom heating increases there is a faster approach toward asymptotic conditions. Evans et al. (Ref. 5) have addressed the effect of non uniform heating on thermal stratification of water inside a rectangular enclosure and shown that thermal stratification can be reduced by distributing the heat flux along the side wall such that the maximum heat flux is concentrated near the bottom and decreases toward the top. The primary mechanism for the reduction of thermal stratification was postulated to be boundary separation at the wall. The bottom of the enclosure (Ref. 5) was insulated while the free interface was coated with a surfactant film of stearic acid to prevent vaporization; though the condition at the free surface is not specified, the effect of the surfactant film may be approximated as an insulating boundary condition based on the asymptotic profile of the rise in temperature of the enclosed fluid.

The experimental works of Evans \& Stefany (Ref. 6) addressed transient heat transfer effects inside totally filled cylindrical enclosures. They show that in response to a step-change in wall temperature, the mixed-mean temperature of the fluid exhibits an exponential rise in temperature which approaches the wall temperature asymptotically. In addition, the heat transfer coefficient oscillates in time as steady-state is approached. This finding was confirmed by the numerical and experimental works of Barakat \& Clark (Ref. 7) for a partially filled cylindrical enclosure. Numerical works have been put forth by Polezhaev 
(Refs. 8 and 9) who considers natural convection inside partially filled cavities for rectangular (Ref. 8) as well as cylindrical (Ref. 9) cavities for various boundary conditions.

In contrast to the above works, our problem is somewhat unique in that there is no free surface and also that the fluid occupy the entire volume of the enclosure. The experimental works of Evans \& Stefany (Ref. 6) share the similarity of total volume filling, however the boundary conditions are different, a stepchange in temperature is prescribed. In our model we consider the Neumann prescribed heat flux conditions along the bottom, top and side of the enclosure. Thus depending on the dominant heat flux condition, there is coupling between natural convection driven by side heating and convective instability driven by bottom heating. The top heating serves as a stabilizing mechanism, due to imposed stable stratification, in contrast to bottom heating in which there is unstable stratification due to an increase of density with height. The dominant mechanism, natural convection or convective instability, depends on the external heat flux conditions during LAD operation. These two mechanism differ from the standpoint that in natural convection problems there is no stability threshold, convection occurs for any temperature variation across the boundary of the cavity whereas convective instability has a stable solution. The characteristics of natural convection inside vertical cavity due to differentially imposed temperature, at the vertical boundary, have been addressed by Elder (Refs. 10 and 11) for laminar (Ref. 10) and turbulent (Ref. 11) free convection. Transition from unicellular to secondary and tertiary flows have been shown to occur during laminar free convection, whereas the turbulent regime is characterized via travelling wavelike motion along the wall. The visual observation by Seki et al. (Ref. 12) has confirmed the findings of Elder, and also demonstrated the effect of Prandtl number. The mechanism of convective instability has been demonstrated for differentially heated horizontal cavity, with a forced flow component, whose bottom temperature is greater than the top by Akiyama et al. (Ref. 13). They show that for the limiting case of forced convection between horizontal plates, the limit of zero Reynolds number (no forced flow) yields agreement with the critical Rayleigh number criterion of 1708 corresponding to the classical Rayleigh-Benard problem (Ref. 14). Secondary flow patterns in the form of longitudinal vortex rolls were observed to occur near the critical Rayleigh number. The two basic configurations (Refs. 10 to 12) of a narrow vertical and horizontal cavity (Ref. 13) serve as basis by which the interaction between natural convection and convective instability mechanism can be understood.

We introduce a model problem which considers the basic physics of heat entrapment inside an enclosure subjected to prescribed heat flux conditions to shed light on the convective state of the flow field in relation to the temperature field. This model problem is intended to lead insight into the temperature measurements (Ref. 1) made for liquid nitrogen inside an enclosure which absorbs heat from ambient laboratory condition, and also to assess the effect of imposed heat flux condition at the bottom boundary. Our main focus is to investigate the effect of heat flow from the ambient laboratory environment to the enclosure in order to establish a baseline to address the effect of imposed heat flux on the bottom boundary. The experiments were conducted using double encapsulation along the sidewall, thus the heat flux absorbed along the sidewall is minimal; we assume a value of heat flow on the order of $1 \mu \mathrm{W}$. However, other unknowns are the heat absorbed on the top and bottom boundary for the experiments carried-out under ambient laboratory conditions. We investigate a range of heat flux condition to lead insight into its effects on the temperature rise of the fluid. This approach is necessary, since the baseline condition is not known and it is needed in order to assess properly the effect of bottom heating.

We show that the effect of heat absorption is to destabilize the equilibrium condition of a fluid inside an enclosure and give rise to flow. The dynamical motion of the flow field obeys the evolutionary equations of mass, momentum and energy. For an incompressible Boussinesq fluid we numerically solve the coupled set of governing equations consisting of the Navier-Stokes coupled with the scalar temperature field using finite-difference methods. We consider the range of Rayleigh numbers (Ra) of order 1 to $10^{9}$ for heating conditions comprising of prescribed heat flux at the sidewall, top, and bottom of the cavity. For the sidewall heat source we show that there is no stability threshold, incipient convection occurs. The flow field is dominated by two counter-rotating cells for low Rayleigh numbers and secondary flows can occur as Ra increases. However, for the bottom heating condition there is a stability threshold for which the onset of convection occurs which is on the order of $10^{5}$. As the Rayleigh number 
increases, the horizontal wavelength which characterizes the onset of convection decreases. There is a transition to asymmetric flow for Ra on the order of $10^{7}$. Direct numerical simulation of the full scale prototype shows that for Ra on the order of $10^{9}$ the onset of convective instability proceeds from short wavelength to nonlinear growth of thermals and culminates with a travelling wave-type mode prior to transition to asymmetry. Due to the large geometric length scale of the prototype, attainment of asymptotic states can be prohibitive. We employ dynamical similarity using a model with smaller geometric dimensions to show that similar dynamical events occur which facilitate the attainment of asymptotic states. As the Rayleigh number increases from $10^{6}$ to $10^{9}$ the flow field undergoes a transition from periodic to chaotic oscillations. The parametric study helps to identify the basic mechanism that drives the flow and lays the foundation to identify the baseline condition for heat entrapment effects.

The paper is organized as follows: we postulate the basic physics of heat entrapment inside an enclosure. We derive the parametric space of the problem through scaling analysis. We solve the coupled set of nonlinear partial differential equations using finite difference methods coupled with a FluxCorrected Transport method to resolve the small scale structure of the temperature field. We show that direct numerical simulation coupled with dynamical similarity can be used advantageously to model the large prototype enclosure used in the experiments. We show the basic convective state of the flow field for a relevant parametric space and that transition to asymmetric flow is relevant to this problem.

\section{Formulation}

The baseline condition for heat entrapment is shown fundamentally in Figure 1A(a) and consists of a cryogenic liquid at saturation condition represented by $\rho_{A}$ which can absorb various amounts of heat at its boundaries comprising of surface heat fluxes $\mathrm{q}_{\mathrm{s}}$, $\mathrm{q}_{\mathrm{t}}$, $\mathrm{q}_{\mathrm{b}}$ " denoting respectively the sidewall, top, and bottom boundaries. The enclosure in Figure 1A can be approximated geometrically as the mid-plane of a cylinder with equivalent height $(\mathrm{H})$ and diameter $(\mathrm{L})$. This basic configuration represents the baseline condition investigated experimentally (Ref. 1) for liquid nitrogen inside a cylindrical Dewar under ambient laboratory condition; for which the heat flux absorb at the sidewall is controlled by external means such as double encapsulation of the wall region to minimize heat flow. Regardless of precautionary measures a finite amount of heat leakage occurred as indicated by the rise of temperature (Ref. 1) of liquid $\rho_{A}$. This finding indicates that under laboratory environment, equilibrium condition is not attained for a given cryogenic liquid $\rho_{A}$. One of the main focus of our model is to investigate the effect of relative magnitude of the heat fluxes $\left(\mathrm{q}_{\mathrm{s}}, \mathrm{q}_{\mathrm{t}}, \mathrm{q}_{\mathrm{b}}\right.$ ) ) on the dynamical state of the liquid $\rho_{A}$ at saturation temperature $\tilde{T}_{A}$ when the system is driven off-equilibrium. This approach is necessary as it eliminates one of the unknown of the problem, if one of the heat flux is prescribed such as $\mathrm{q}_{\mathrm{b}}$ " in the experiments (Ref. 1). Thus for a given q", optimal values for $\mathrm{q}_{\mathrm{s}}$ " and $\mathrm{q}_{\mathrm{t}}$ "can be estimated based on ambient condition and experimental arrangement. Given the double encapsulation used on the sidewall of the experiments (Ref. 1), it is approximated that $\mathrm{q}_{\mathrm{s}}$ " $<<\mathrm{q}_{\mathrm{t}}$. Hence for the baseline condition the dominant heat fluxes are $\mathrm{q}_{\mathrm{b}}$ " and $\mathrm{q}_{\mathrm{t}}$.

More generally the effect of heat entrapment is investigated experimentally (Ref. 1) by placing a divider at mid-height, consisting of stainless-steel screen meshes or an aluminum solid divider, of the cavity as shown in Figure $1 \mathrm{~A}(\mathrm{~b})$ in which $\rho_{B}=\rho_{A}$. The effect of heat entrapment is quantified experimentally by measuring the temperature above and below the divider which is used to compare against the baseline experiment illustrated in Figure 1A(a). The findings (Ref. 1) indicate that temperatures below (bottom)/above (top) the divider position were higher/lower with the divider installed indicating heat entrapment/reduced stratification. The effects of the divider may be quantified by approximating the local heat flux $\mathrm{q}_{\mathrm{i}}$ " into or out of the top region. Hence the problem may be simplified into two sub-problems as in Figure 1B in which heat flows into or out of the top/bottom region shown in (a) and (b). This simplification effectively reduces the aspect ratio by $1 / 2$ and the problem may be similarly addressed as the baseline problem in Figure 1A(a) in which $\mathrm{q}_{\mathrm{b}}$ " and $\mathrm{q}_{\mathrm{t}}$ " can take the respective values of $\mathrm{q}_{\mathrm{i}}$. This approach effectively reduces the parametric range of the problem which can be used to investigate the more general set of conditions as represented in Figure 1A(b). 

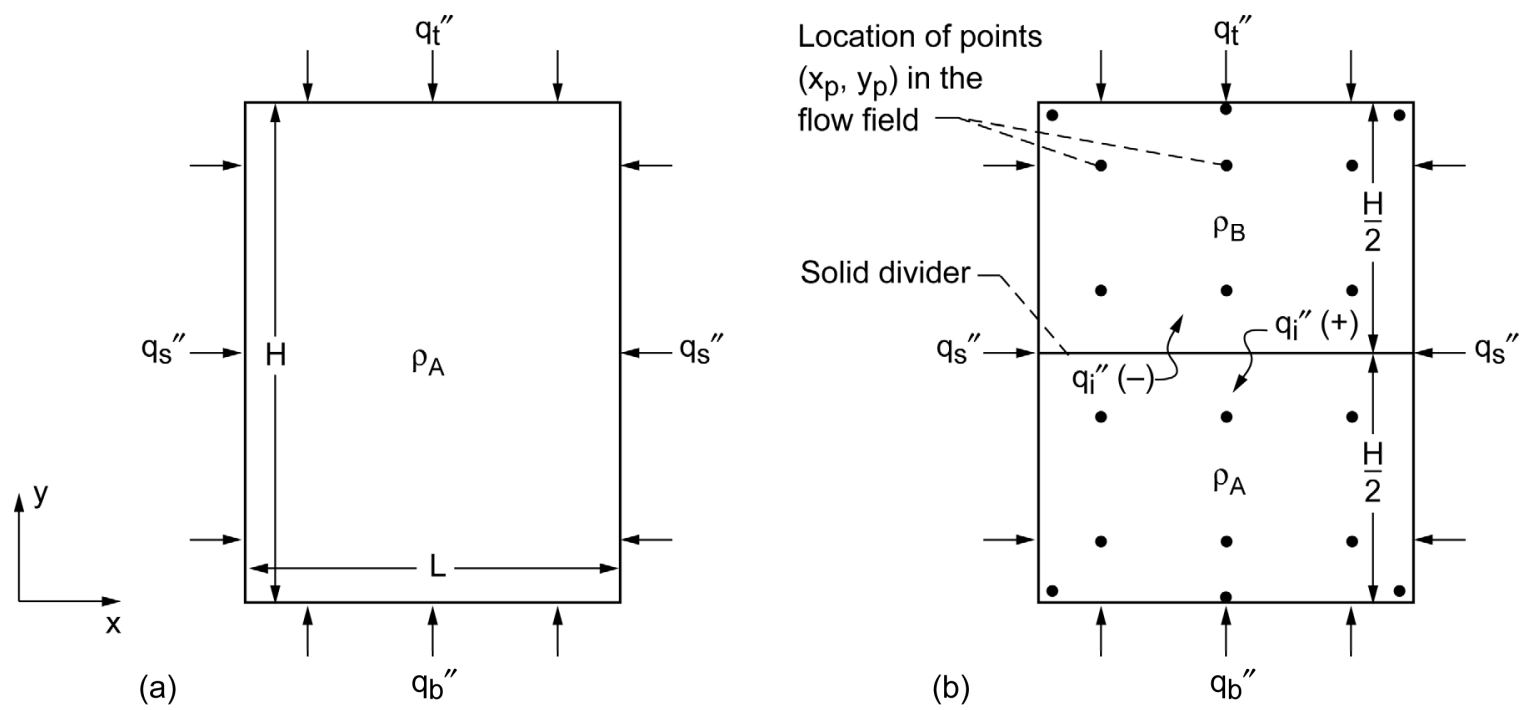

Figure 1A.-Physical description of heat entrapment concept showing heat flux absorption $\left(q_{b}{ }^{\prime \prime}, q_{s}{ }^{\prime \prime}, q_{t}{ }^{\prime \prime}\right)$ into cryogenic fluid $\left(\rho_{A}\right)(a)$ without a solid divider and $(b)$ with a solid divider which admits heat flux $q_{i}{ }^{\prime \prime}(-)$ into or out $q_{i}^{\prime \prime}(+)$ of the top region $\left(\rho_{B}\right)$.

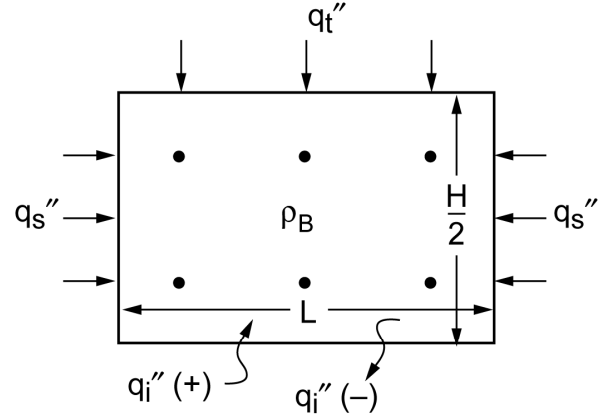

(a)

Figure 1B.-Subproblem of enclosure with solid divider showing (a) top region and (b) bottom region.

In general, as shown in Figure $1 \mathrm{~A}(\mathrm{a})$, a condition for equilibrium to exist is for $\mathrm{q}_{\mathrm{s}} "=\mathrm{q}_{\mathrm{t}}$ " $=\mathrm{q}_{\mathrm{b}}$ " $=0$; this condition is not likely met for a low temperature cryogenic liquid inside an enclosure. Thus offequilibrium condition need to satisfy the dynamical motion of the fluid govern by the continuity, momentum and energy equations as:

$$
\begin{gathered}
\frac{\partial u}{\partial x}+\frac{\partial v}{\partial y}=0 \\
\frac{\partial u}{\partial t}+u \frac{\partial u}{\partial x}+v \frac{\partial u}{\partial y}=-\frac{\partial p}{\partial x}+\nu\left(\frac{\partial^{2} u}{\partial x^{2}}+\frac{\partial^{2} v}{\partial y^{2}}\right) \\
\frac{\partial v}{\partial t}+u \frac{\partial v}{\partial x}+v \frac{\partial v}{\partial y}=-\frac{\partial p}{\partial y}+\nu\left(\frac{\partial^{2} v}{\partial x^{2}}+\frac{\partial^{2} v}{\partial y^{2}}\right)-\beta \Delta \tilde{T} n g_{0}
\end{gathered}
$$




$$
\frac{\partial \tilde{T}}{\partial t}+u \frac{\partial \tilde{T}}{\partial x}+v \frac{\partial \tilde{T}}{\partial y}=\alpha\left(\frac{\partial^{2} \tilde{T}}{\partial x^{2}}+\frac{\partial^{2} \tilde{T}}{\partial y^{2}}\right)
$$

In the above equations the density field is independent of pressure due to incompressibility of the liquid, however it is a function of temperature and can be linearized and given as an equation of state,

$$
\rho=\rho\left(\tilde{T}_{A}\right)[1-\beta \Delta \tilde{T}]
$$

We consider a Boussinesq fluid in which variation of density is significant only in the body force term as shown in Equation (3) where the coefficient of thermal expansivity is given as $\beta=-1 / \rho(\partial \rho / \partial \tilde{T})_{P}$.

Superscript $\sim$ denotes dimensional temperature and $\Delta \tilde{T}=\tilde{T}-\tilde{T}_{A}$. The thermophysical properties of the liquid, its density $\rho$, kinematic viscosity $v$, and thermal diffusivity $\alpha$ are taken to be at saturation condition for the specific cryogen, for liquid nitrogen $\tilde{T}_{A}=77^{\circ} \mathrm{K}$. The gravitational acceleration $\mathrm{g}_{\mathrm{y}}$ is denoted by $\mathrm{ng}_{0}$ in which $\mathrm{n}$ is a ratio by which the standard gravitational acceleration $\mathrm{g}_{\mathrm{o}}$ can be reduced via low gravitational environments such as the International Space Station (ISS), Moon, and Mars surface.

The description of the initial and boundary conditions are made in reference to Figure 1A(a). The initial condition is taken as uniform temperature throughout the liquid,

$$
t=0, \tilde{T}(x, y, 0)=\tilde{T}_{A}
$$

The momentum Equations (2) and (3) satisfy the no-slip condition along the boundary of the cavity denoted by $\Gamma$ and given as,

$$
u(x, y, t)=0, v(x, y, t)=0 \text { on } \Gamma
$$

Whereas the energy Equation (4) satisfy the Neumann condition along the boundary $\Gamma$ prescribed as,

$$
\begin{aligned}
& \left.x=0, q^{\prime \prime}=q_{s} ", 0 \leq y \leq H, \partial \tilde{T} / \partial x\right)_{x=0}=-q_{s} " / k \\
& \left.x=L, q^{\prime \prime}=q_{s} ", 0 \leq y \leq H, \partial \tilde{T} / \partial x\right)_{x=L}=-q_{s} " / k \\
& \left.y=0, q^{\prime \prime}=q_{b}, 0 \leq x \leq L, \partial \tilde{T} / \partial y\right)_{y=0}=-q_{b} " / k \\
& \left.y=H, q^{\prime \prime}=q_{t}^{\prime \prime}, 0 \leq x \leq L, \partial \tilde{T} / \partial y\right)_{y=H}=-q_{t}^{\prime \prime} / k
\end{aligned}
$$

\section{Scaling Analysis and Computational Method}

The governing set of Equations (1) to (4) may be simplified by transforming the momentum Equations (2) and (3) into a vorticity equation using the following definition,

$$
u=\frac{\partial \psi}{\partial y}, v=-\frac{\partial \psi}{\partial x}, \quad \xi=\frac{\partial v}{\partial x}-\frac{\partial u}{\partial y}
$$


The resulting set of equations is scaled using the characteristics length, time, and velocity as $\left(L, L^{2} / \alpha, \alpha / L\right)$, respectively. The dimensionless temperature is scaled as

$$
T=\frac{\tilde{T}-\tilde{T}_{A}}{q " L / k}
$$

The scaled dimensionless (denoted by *) set of equations become,

$$
\begin{gathered}
\frac{\partial^{2} \psi^{*}}{\partial x^{* 2}}+\frac{\partial^{2} \psi^{*}}{\partial y^{* 2}}=-\zeta^{*} \\
\frac{\partial \xi^{*}}{\partial t^{*}}+\left(u^{*} \frac{\partial \xi^{*}}{\partial x^{*}}+v^{*} \frac{\partial \xi^{*}}{\partial y^{*}}\right)=\operatorname{Pr}\left(\frac{\partial^{2} \xi^{*}}{\partial x^{* 2}}+\frac{\partial^{2} \xi^{*}}{\partial y^{* 2}}\right)+R a \operatorname{Pr} \frac{\partial T}{\partial x^{*}} \\
\frac{\partial T}{\partial t^{*}}+\left(u^{*} \frac{\partial T}{\partial x^{*}}+v^{*} \frac{\partial T}{\partial y^{*}}\right)=\left(\frac{\partial^{2} T}{\partial x^{* 2}}+\frac{\partial^{2} T}{\partial y^{* 2}}\right)
\end{gathered}
$$

The boundary conditions become,

$$
\begin{aligned}
& x^{*}=0,0 \leq y^{*} \leq A r, \partial T / \partial x^{*} x^{*}=0=-f_{s} \\
& x^{*}=1,0 \leq y^{*} \leq A r, \partial T / \partial x^{*} x^{*}=1=-f_{s} \\
& \left.y^{*}=0,0 \leq x^{*} \leq 1, \partial T / \partial y^{*}\right)_{y}^{*}=0=-f_{b} \\
& \left.y^{*}=A r, 0 \leq x^{*} \leq 1, \partial T / \partial y^{*}\right)^{*}=A r=-f_{t}
\end{aligned}
$$

The set of parameters resulting from scaling is defined as,

$$
R a=\frac{\beta q^{\prime \prime} n g_{o} L^{4}}{k \nu \alpha}, \operatorname{Pr}=\frac{\nu}{\alpha}, A r=\frac{H}{L}, f_{s}=\frac{q_{s}^{\prime \prime}}{q^{\prime \prime}}, f_{b}=\frac{q_{b}^{\prime \prime}}{q^{\prime \prime}}, f_{t}=\frac{q_{t}^{\prime \prime}}{q^{\prime \prime}}
$$

which represents respectively the Rayleigh and Prandtl numbers, aspect ratio, and dimensionless heat fluxes. Thus the parametric set,

$$
\Lambda=\Lambda\left(R a, A r, \operatorname{Pr}, f_{s}, f_{b}, f_{t}\right)
$$

consists of six parameters that can be varied independently and represents a formidable parametric set. Simplification of the parametric range is obtained by addressing the relevant limiting conditions for a given aspect ratio, fixed cryogenic liquid, and typical heat flux scale q". Since the predominant control variable is the bottom heat flux q $\mathrm{q}_{\mathrm{b}}$ " which implies $f_{b}=1$, then $f_{s}, f_{t}$ can take a range of values from zero to one. The conditions $f_{t}=0$ and $f_{t}=1$ signify insulated and heat absorption top boundary conditions, respectively. The side conditions $f_{s}=0$ and $f_{s}=1$ indicate a perfectly insulated sidewall and dominant sidewall heating. Since the sidewall has double encapsulation in the experiments (Ref. 1), we consider 
values of $f_{s}$ on the order of $10^{-6}$ which is approximately $1 \mu \mathrm{W}$ of heat leakage. Although this heat leakage is insignificant in comparison to $f_{b}$ and $f_{t}$, it serves as a perturbation parameter to excite flow instability.

The governing set of Equations (14) to (16) with boundary conditions (Eqs. (7) and (17) to (20)) are solved using finite-difference methods with a direct solver approach. The solution methodology consists of solving the stream function Poisson's Equation (14) using matrix inversion which is followed by the solution of the energy Equation (16) and vorticity Equation (15). The vorticity field is used subsequently for each time step. Since we are interested in time evolution of the flow field dynamics, a time accurate methodology is employed which uses third order Adams Bashforth for time discretization. Since the bottom heat flux is dominant, the convective instability mechanism becomes important in comparison to natural convection, which requires high resolution of the temperature field. We employed the Flux Corrected Transport (FCT) method (Ref. 15) to resolve sharp interior gradients; in addition this method eliminates false numerical oscillation by ensuring positivity of the temperature scalar component throughout the domain of computation.

\section{Numerical Results}

\section{Stabilization of Flow Field}

The dynamical state of fluid motion inside the enclosure shown in Figure 1A(a) is first investigated for ideal cases in order to show the fundamental mechanism of convective motion. We first consider how stable states can be obtained and the cause of departures from stability. This is illustrated using liquid hydrogen as an example with Prandtl number $\operatorname{Pr}=1.03$. The destabilization of the flow field for bottom and side heat flux is summarized in Figure 2. The heating prescriptions that lead to convective stability in which there is no fluid motion even though conduction dominates is illustrated for $\mathrm{Ra}=1.26 \times 10^{3}$ in Figure 2(a). These trends show that stability of the flow field can be obtained, below the critical Rayleigh number, provided that there is a perfectly insulated sidewall $f_{s}=0$ for cases in which: (1) there is bottom heating only $\left(f_{b}=1, f_{t}=0\right),(2)$ top heating only $\left(f_{b}=0, f_{t}=1\right)$, and (3) both top and bottom absorbing heat $\left(f_{b}=1, f_{t}=1\right)$. The case when $\left(f_{b}=1, f_{t}=1\right)$ represents actual experimental condition, since neither top $f_{t}=1$ nor bottom heating $f_{b}=1$ can be isolated. However these cases represent idealization of an experimental system (Ref. 1) since there is finite heat leakage along the sidewall, i.e., $f_{s}>0$, regardless of precautionary measures. Note that absolute stability occurs for case 2 regardless of the Rayleigh number magnitude, however, at a critical Rayleigh number cases 1 and 3 become unstable marked by the onset of convection.

\section{Perturbation of Stability}

The perturbation of the stability for bottom $\left(f_{b}=1, f_{t}=0\right)$ and top heating $\left(f_{b}=0, f_{t}=1\right)$ is illustrated in Figure 2(b) for $\mathrm{Ra}=1.26 \times 10^{6}$ in order to isolate simultaneous effects of bottom and top heating, and to show the interaction of natural convection and convective instability. For a conservative heat leakage on the order of $1 \mu \mathrm{W}$ projected on the sidewall surface of a cylinder $f_{s}=2 \times 10^{-6}$ (qs" $=0.001 \mathrm{erg} / \mathrm{cm}^{2}-\mathrm{s}$ ), the stable base state $\left(f_{b}=1, f_{t}=0\right)$ becomes unstable due to unstable stratification caused by heavy fluid overlaying lighter fluid due to decrease of local density near the bottom of the enclosure caused by bottom heating $\left(f_{b}=1\right)$. This case corresponds to $\mathrm{q}_{\mathrm{b}} "=500 \mathrm{erg} / \mathrm{cm}^{2}-\mathrm{s}$ which can be obtained from heat flow from ambient laboratory environment on the order of $10 \mathrm{~mW}$. In this case convective instability driven by bottom heating is dominant over natural convection driven by side heating. Since we are operating near the threshold of instability the growth of four modes occur. In contrast to instability of the bottom stratification $\left(f_{b}=1, f_{t}=0, f_{s}=0\right)$, with a side heat leakage of $f_{s}=2 \times 10^{-6}$, we show that the top stratified layer $\left(f_{b}=0, f_{t}=1, f_{s}=0\right)$ remains stable for the same heat leakage; however horizontal density gradient gives rise to a natural convection base flow with a symmetric double cell. These two cases illustrate that even though the top stratified layer is stable for all Rayleigh numbers when the sidewall is insulated $f_{s}=0$, the effect of finite side heat leakage is to drive a natural convection base flow. 
(a) $\mathrm{Ra}=1.26 \times 10^{3}$ (stabilization of flow field)
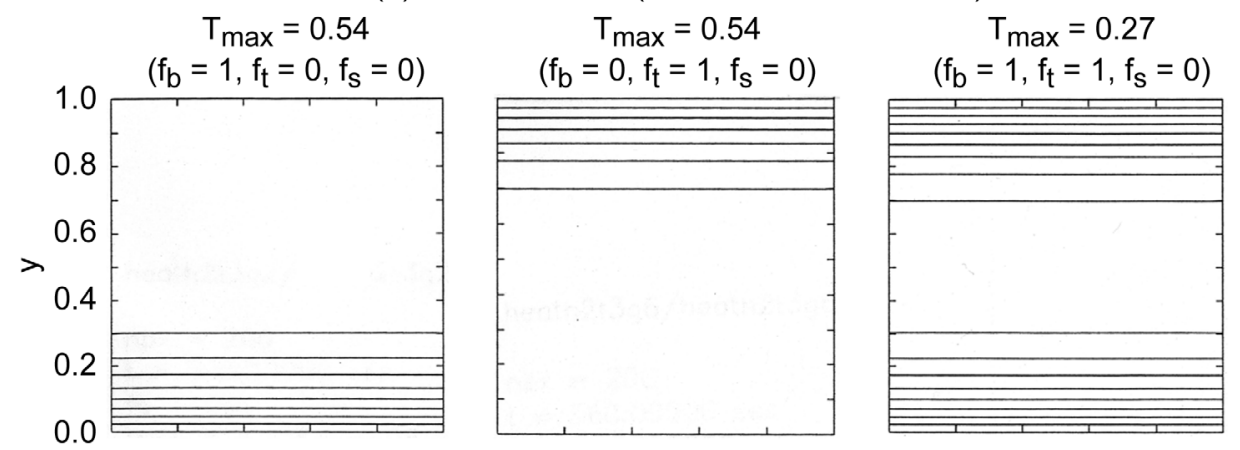

(b) $\mathrm{Ra}=1.26 \times 10^{6}$ (perturbation of stability)

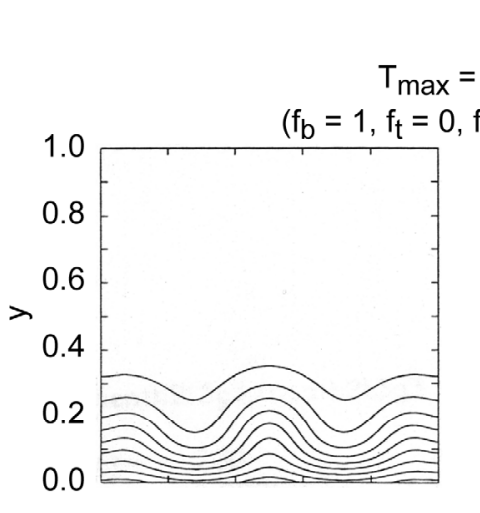

$\max =0.57$
$\left.=0, f_{S}=2 \times 10^{-6}\right)$

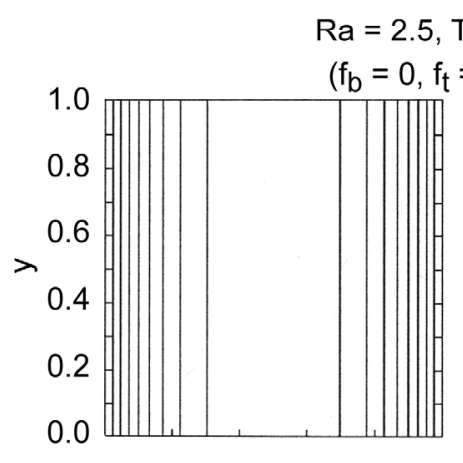

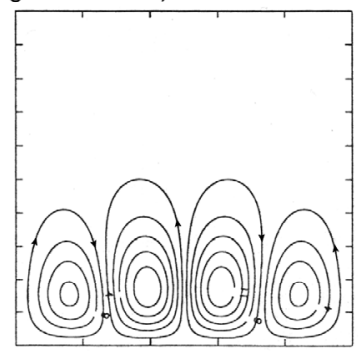

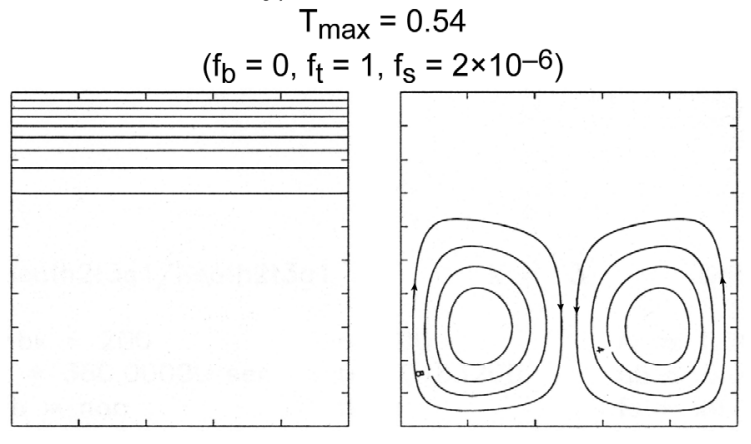

(c) (Effect of side heating)

$\mathrm{Ra}=1.26 \times 10^{6}, \mathrm{~T}_{\max }=0.54$

$\left(\mathrm{f}_{\mathrm{b}}=0, \mathrm{f}_{\mathrm{t}}=0, \mathrm{f}_{\mathrm{s}}=1\right)$
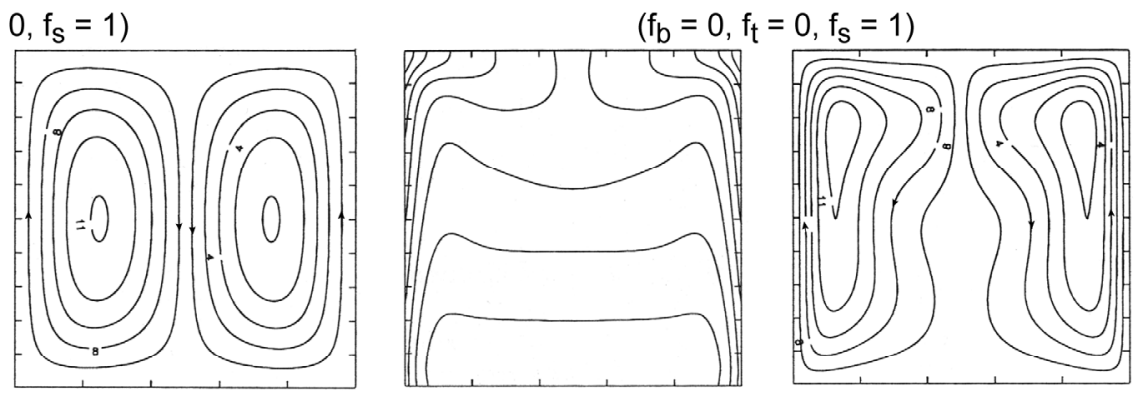

(d) $\mathrm{Ra}=1.26 \times 10^{6}$ (perturbation of double layer)

$\mathrm{T}_{\max }=0.29$

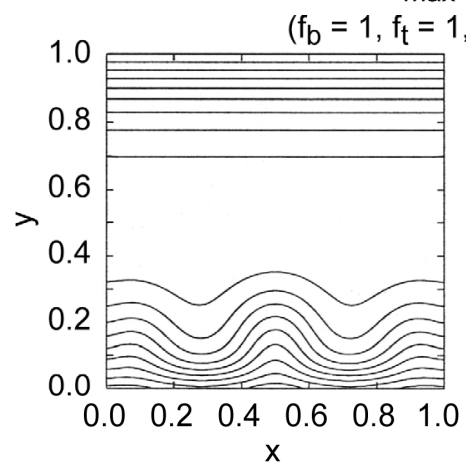

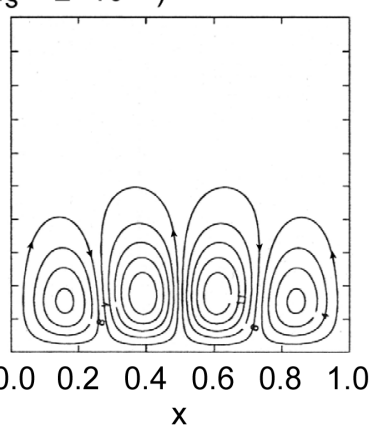

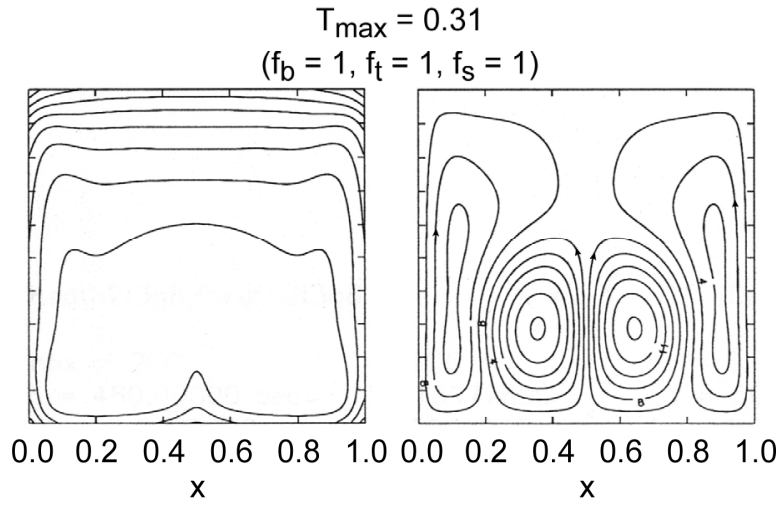

Figure 2.-Destabilization of flow field due bottom and side heat flux $(t=360 \mathrm{~s}) ; \mathrm{qb}^{\prime \prime}, \mathrm{qt}_{\mathrm{t}}{ }^{\prime}$ are nominally $500 \mathrm{erg} / \mathrm{cm}^{2}-\mathrm{s}$, $\mathrm{qs}^{\prime \prime}=0.001 \mathrm{erg} / \mathrm{cm}^{2}-\mathrm{s}$ for side heat flux perturbation. 


\section{Effect of Side Heating}

Since side heat leakage is an effective source to excite instability and drive a natural convective base flow, we isolate the effect of side heating by considering the case when there is no heat leakage from the bottom $\left(f_{b}=0\right)$ and top $\left(f_{t}=0\right)$ contrasting $\mathrm{Ra}=2.5$ and $\mathrm{Ra}=1.26 \times 10^{6}$ in Figure 2(c). These two cases contrast heat flow on the order of $1 \mu W\left(\mathrm{qs} "=0.001 \mathrm{erg} / \mathrm{cm}^{2}\right.$-s) and $10 \mathrm{~mW}\left(\mathrm{qs} "=500 \mathrm{erg} / \mathrm{cm}^{2}-\mathrm{s}\right)$ corresponding to $\mathrm{Ra}=2.5$ and $\mathrm{Ra}=1.26 \times 10^{6}$, respectively. For $\mathrm{Ra}=2.5$ natural convection becomes dominant, it generates a very weak flow field which does not disturb the conduction layer near the vertical walls. However, when there is significant side heat leakage, $\mathrm{Ra}=1.26 \times 10^{6}$, the cellular structure becomes unstable which results in growth of impending secondary modes due to shear instability. There is a transition from conduction dominated regime to a boundary layer regime indicated by the temperature field. The flow field transports warm fluid near the walls to the interface region which forms a stratified layer. The scenario of sidewall dominated heating is similar to events that occur in applications to address the effect of thermal stratification for partially filled cryogenic storage tanks (Refs. 8 and 9). The case for $\mathrm{Ra}=1.26 \times 10^{6}$ $\left(f_{b}=0, f_{t}=0\right)$ is demonstrated for illustrative purposes; the precautionary measures taken to insulate the sidewall in the experiments (Ref. 1) would indicate a much smaller side heat leakage toward the lower limit of $\mathrm{Ra}=2.5$.

\section{Perturbation of Double Layer}

A typical heating condition with finite sidewall heating is illustrated in Figure 2(d) for the case when there is simultaneous heat absorption from the top and bottom boundaries which represents perturbation of the double layer for $\mathrm{Ra}=1.26 \times 10^{6}$ based on case 3 . The destabilization of the conduction dominated regime $\left(f_{b}=1, f_{t}=1, f_{s}=0\right)$, near the critical Rayleigh number, is contrasted for $f_{s}=2 \times 10^{-6}$ and $f_{s}=1$. When $f_{s}=2 \times 10^{-6}$ convective instability dominates, there occurs growth of four modes at the bottom region while the growth of the stratified layer at the top region for early time $t=360 \mathrm{~s}$ remains stable. However, if there is significant sidewall heat leakage $\left(f_{b}=1, f_{t}=1, f_{s}=1\right)$ natural convection dominates and the characteristic of convection switches from an instability mechanism to a boundary layer dominated flow shown by the convective cells along the wall and secondary cells near the bottom. In this case the intensity of convection modifies the top stratified layer and there is a potential for local superheating near the top corners. In summary, the mechanism by which the double layer $\left(f_{b}=1, f_{t}=1\right.$, $f_{s}=0$ ) becomes unstable is illustrated by perturbation of the stability with $f_{s}=2 \times 10^{-6}$, and the effect of dominant side heating on the stability is illustrated for $f_{s}=1$. This indicates that there can be a wide range of dynamical events that occurs for $2 \times 10^{-6}<f_{s}<1$, even though we fix $f_{s}=2 \times 10^{-6}$ for the parametric study, it is a key parameter to characterize heat entrapment effects. The results also show that when the double layer is perturbed convective mixing becomes important. These limiting conditions serve to illustrate the interaction of convective instability and natural convection that can occur for the baseline condition shown in Figure 1A(a).

\section{Mechanism of Instability}

For the given experimental conditions, it is likely that the convective characteristic inside the enclosure is dominated by convective instability $\left(f_{s}=2 \times 10^{-6}\right)$. In Figure $3 \mathrm{~A}$ we illustrate the mechanism of the instability as the Rayleigh number increases. The results show that from the threshold of instability $\mathrm{Ra}=1.26 \times 10^{5}$ there is a transition from long to short wavelength as Ra increases to $1.26 \times 10^{9}$; there is saturation in mode numbers between $\mathrm{Ra}=1.26 \times 10^{7}$ and $\mathrm{Ra}=1.26 \times 10^{9}$. The growth of modes segregates near the walls as Ra increases and the wavelength between the cells decreases. This trend indicates that high Rayleigh number flows require resolution of small scales for computational accuracy. 


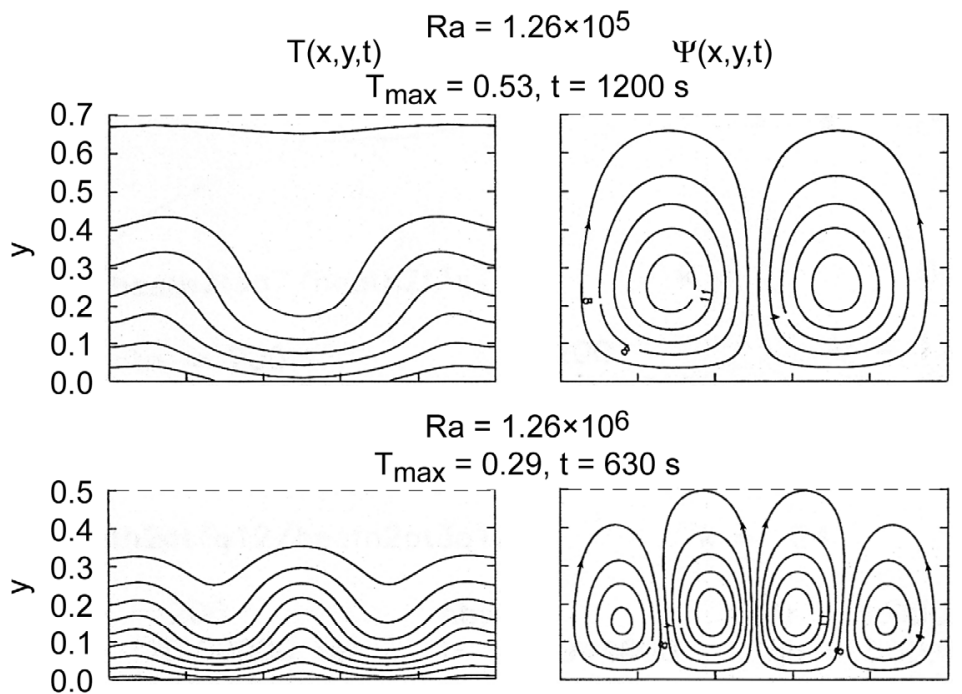

$\mathrm{Ra}=1.26 \times 10^{7}$

$\mathrm{T}_{\max }=0.17, \mathrm{t}=120 \mathrm{~s}$

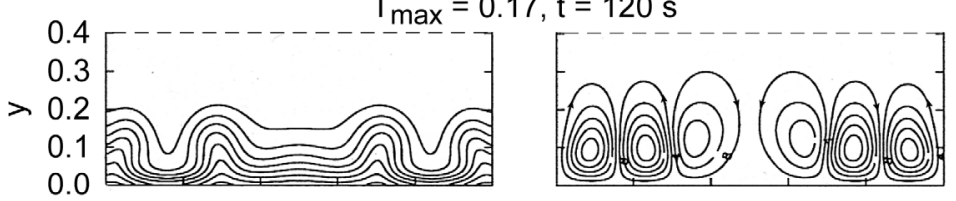

$\mathrm{Ra}=1.26 \times 10^{8}$

$T_{\text {max }}=0.09, \mathrm{t}=38 \mathrm{~s}$

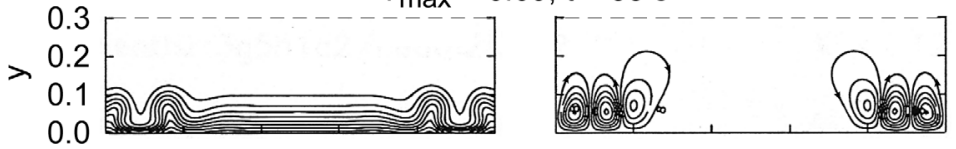

$\mathrm{Ra}=1.26 \times 10^{9}$

$\mathrm{T}_{\max }=0.05, \mathrm{t}=12 \mathrm{~s}$

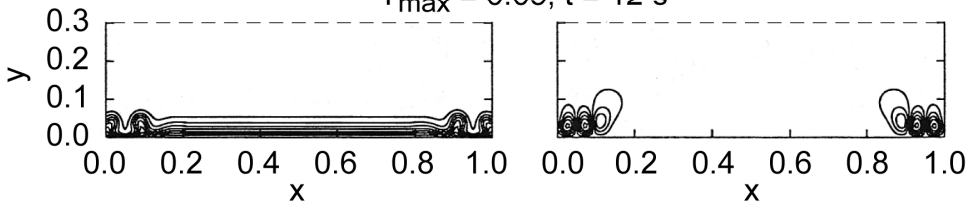

Figure 3A.-Transition to short wavelength instability as Ra increases $\left(f_{b}=1, f_{t}=1, f_{s}=2 \times 10^{-6}\right) q^{\prime \prime}=500 \mathrm{erg} / \mathrm{cm}^{2}-\mathrm{s}, \operatorname{Ar}=1, \operatorname{Pr}=1.03$. 


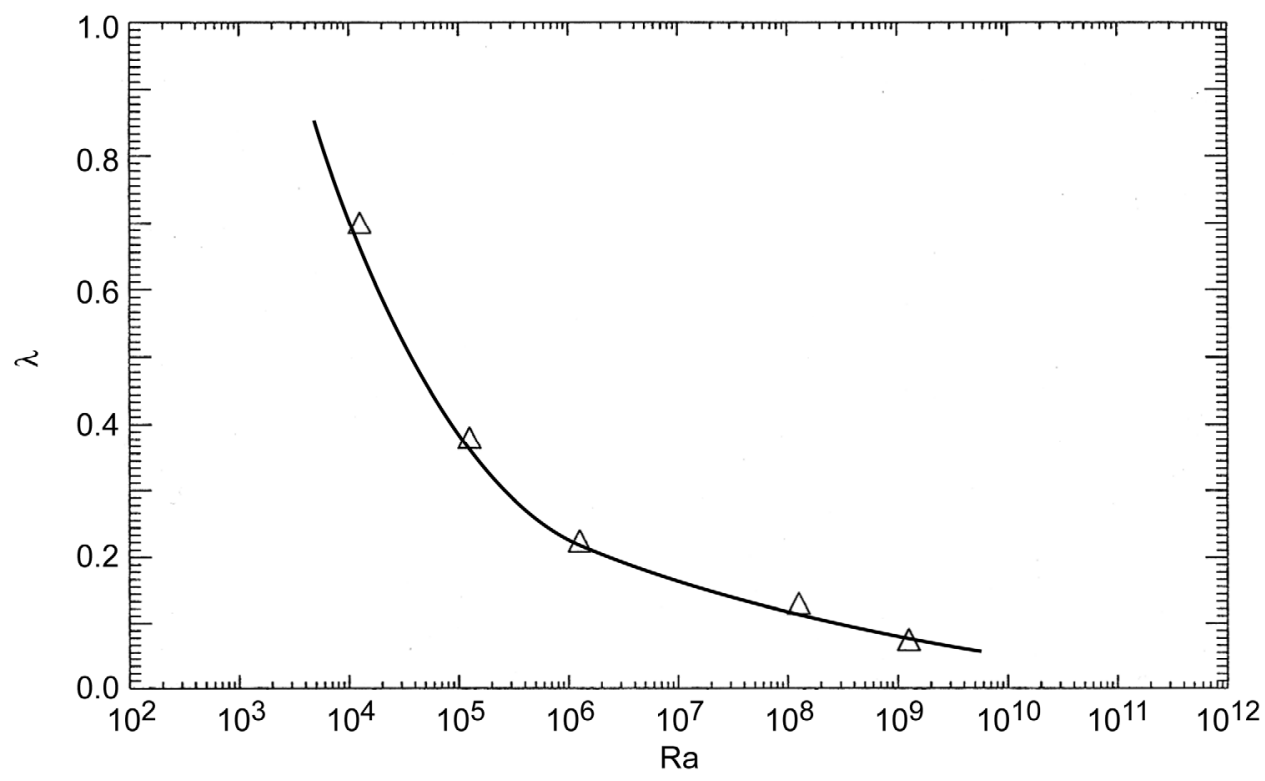

Figure 3B.-Horizontal wavelength of flow field as a function of $R a\left(f_{b}=1, f_{t}=1\right.$, $\left.f_{S}=2.0 \times 10^{-6}\right) \operatorname{Ar}=1, \operatorname{Pr}=1.03$.

The characteristic wavelength $(\lambda=\lambda * / L)$ as a function of the Rayleigh number in Figure 3B shows that $\lambda$ is inversely proportional to $\mathrm{Ra}$, thus decreases sharply as $\mathrm{Ra}$ increases. For the highest Rayleigh number considered $\left(1.26 \times 10^{9}\right), \lambda=0.075$ which is on the order of $4 \mathrm{~mm}$. This finding indicates that the length scale which needs to be resolved gets smaller and smaller for $\mathrm{Ra}>10^{9}$ and also illustrates the challenge posed for computations of high Rayleigh numbers.

\section{Dynamical Events}

We now turn to the dynamical events as the flow field approaches asymptotic states. This is illustrated for $\mathrm{Ra}=1.26 \times 10^{6}$ in Figure 4, which shows growth of a single thermal, and $\mathrm{Ra}=1.26 \times 10^{7}$ in Figure 5A which shows growth of double thermals and the breakdown of symmetry in Figure 5B. In contrast to natural convection dominated flows in which the maximum temperature occurs near the sidewalls due to side heating (Fig. 2(c)), in this convective instability dominated situation (Fig. 4), the maximum temperature occurs near the mid-center of the cavity due to bottom heating since the sidewalls are nearly insulated $\left(f_{s}=2 \times 10^{-5}\right)$. The mechanism of the instability is due to the release of potential energy from unstable stratification caused by heavy fluid overlaying lighter fluid and its conversion to kinetic energy to drive fluid motion. In this case, the warmer fluid transported upwards and cooler fluid moving downwards result in the release of potential energy; the inner cells grow faster than the outer cells since the inner region has the maximum temperature. The inner cells grow as time increases, thus the instability self propagates until the top wall is reached. The initial bottom four cells $(t=336 \mathrm{~s})$ selforganizes into two rows of secondary cells $(t=408 \mathrm{~s})$ due to the nonlinear growth of the thermal. Since the top of the cavity is insulated $\left(f_{t}=0\right)$, the intensity of convection increases as the top of the cavity is approached. Buoyancy effects due to growth of the thermal propel the two inner cells toward the top of the cavity $(t=360 \mathrm{~s})$ while the outer cells near the walls segregate toward the center of the cavity $(t=384 \mathrm{~s})$. The maximum temperature decreases with time due to local mixing induced by the increase in convective intensity for $\mathrm{Ra}=1.26 \times 10^{6}$. 

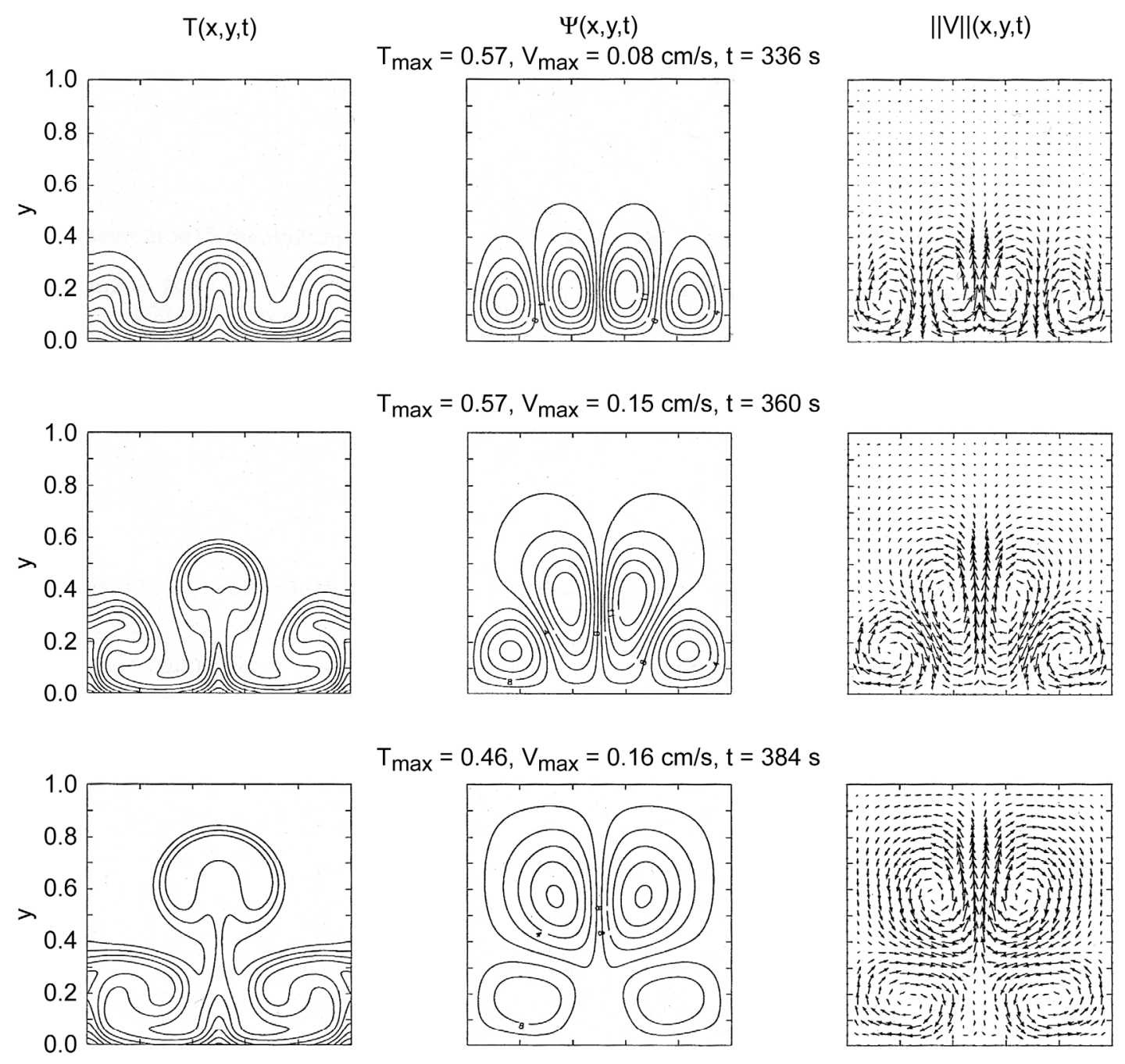

$\mathrm{T}_{\max }=0.46, \mathrm{~V}_{\max }=0.16 \mathrm{~cm} / \mathrm{s}, \mathrm{t}=384 \mathrm{~s}$
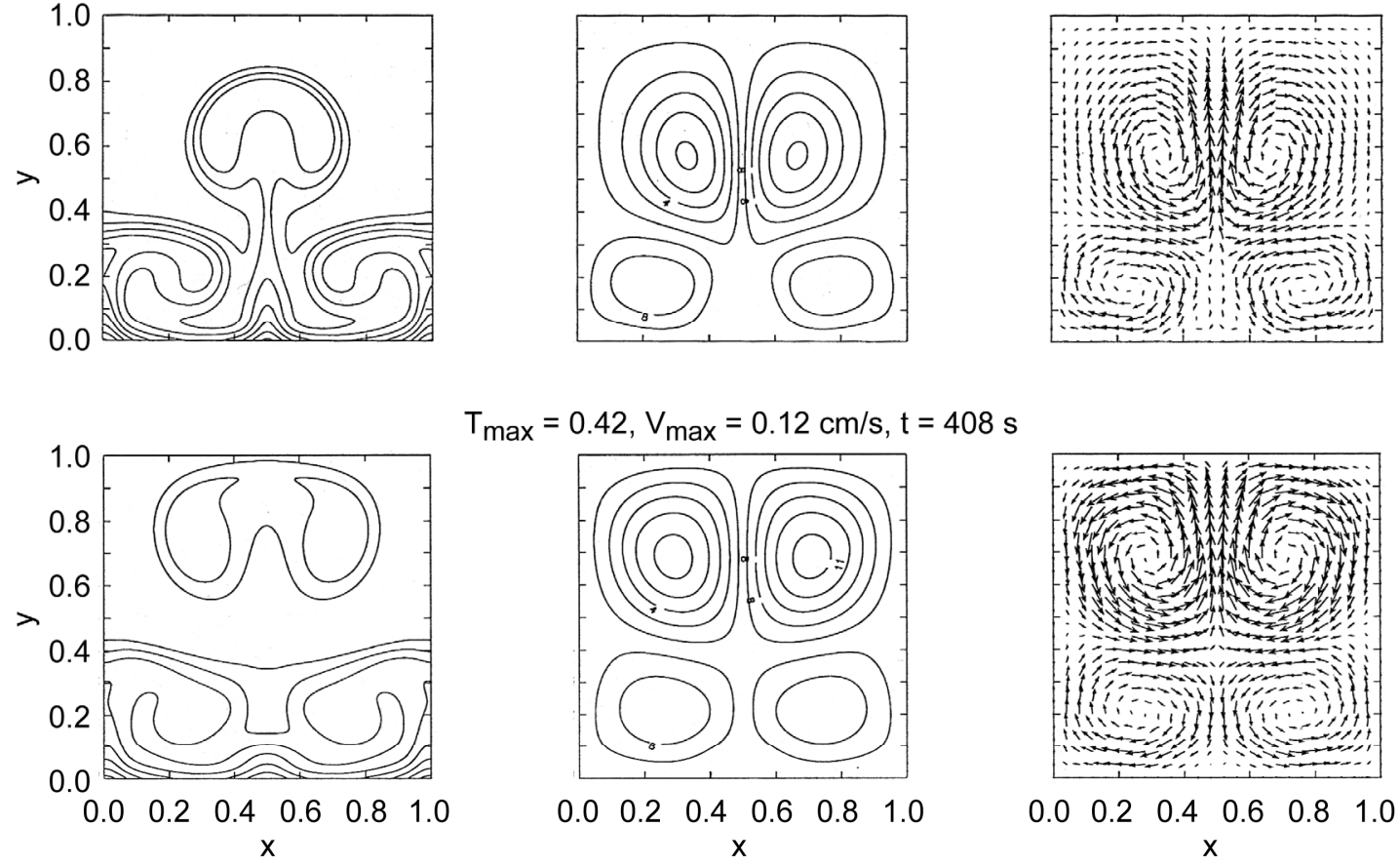

Figure 4.-Evolution of thermal due to nonlinear growth of 4 modes, $\left(f_{b}=1, f_{t}=0, f_{s}=2 \times 10^{-5}\right)$, $\operatorname{Ra}=1.26 \times 10^{6}, \operatorname{Ar}=1, \operatorname{Pr}=1.03$. 

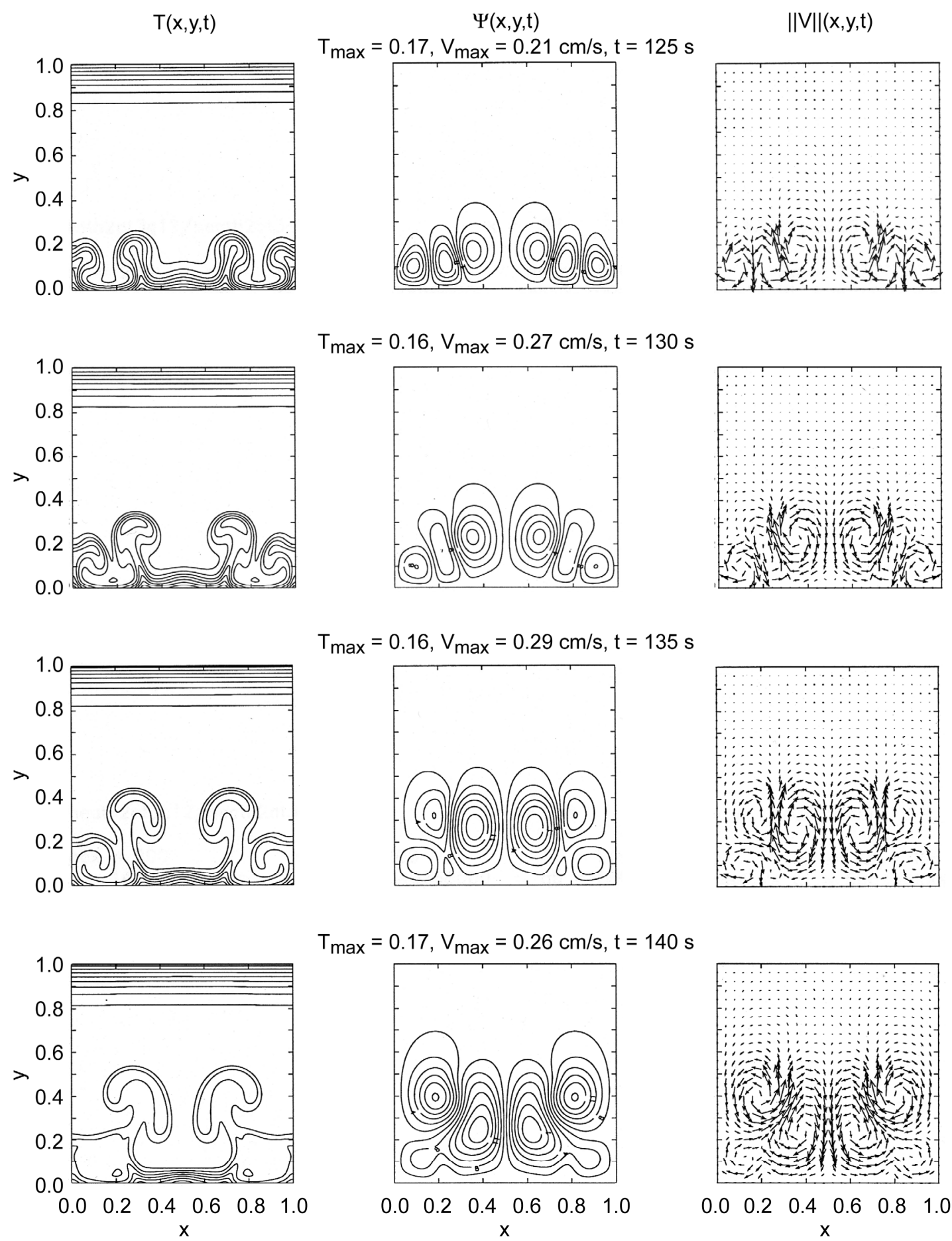

Figure 5A.-Evolution of thermals due to nonlinear growth of 6 modes, $\left(f_{b}=1, f_{t}=1, f_{s}=2 \times 10^{-6}\right)$, $\operatorname{Ra}=1.26 \times 10^{7}, \operatorname{Ar}=1, \operatorname{Pr}=1.03$. 

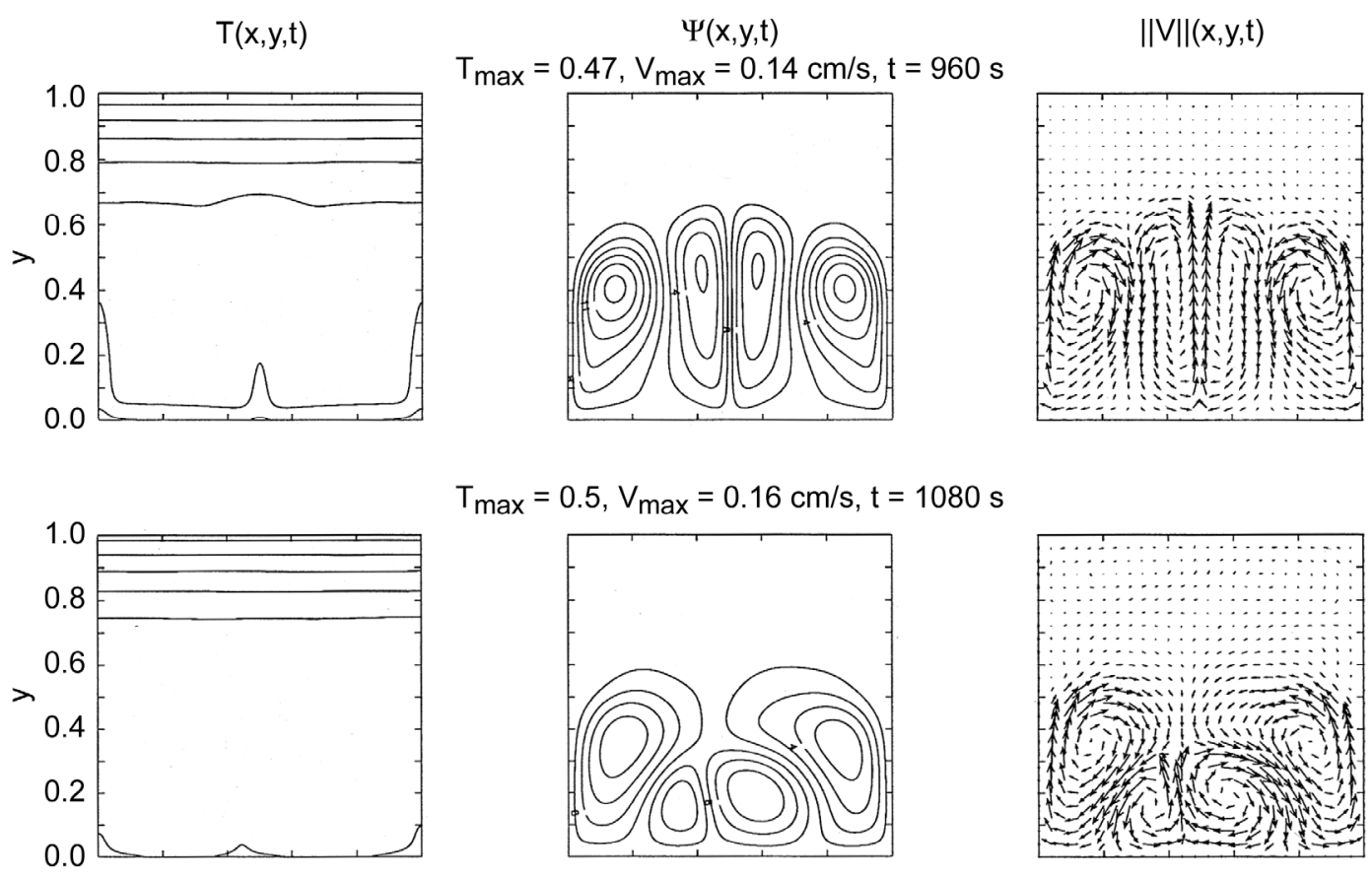

$\mathrm{T}_{\max }=0.5, \mathrm{~V}_{\max }=0.16 \mathrm{~cm} / \mathrm{s}, \mathrm{t}=1080 \mathrm{~s}$
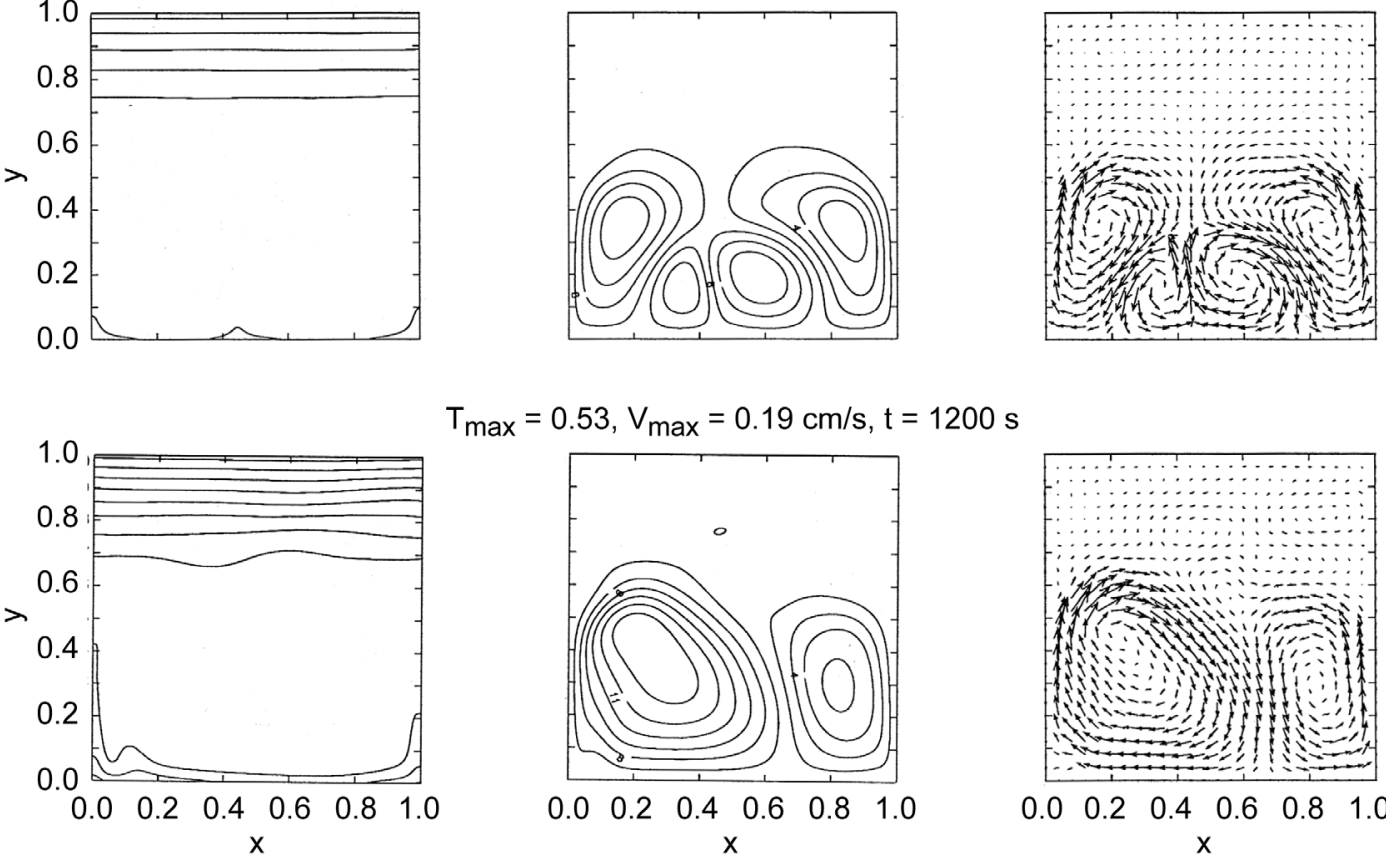

$\mathrm{T}_{\text {max }}=0.53, \mathrm{~V}_{\max }=0.19 \mathrm{~cm} / \mathrm{s}, \mathrm{t}=1200 \mathrm{~s}$
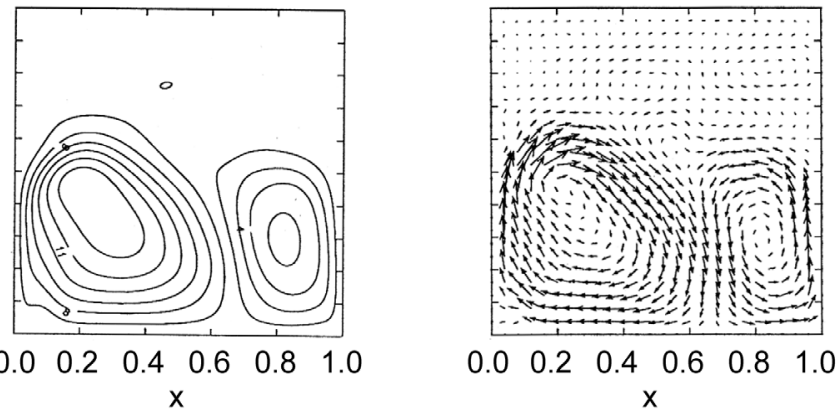

Figure 5B.-Transition to asymmetric flow, $\left(f_{b}=1, f_{t}=1, f_{s}=2 \times 10^{-6}\right), \operatorname{Ra}=1.26 \times 10^{7}, \operatorname{Ar}=1, \operatorname{Pr}=1.03$.

We consider the dynamical evolution of the flow field when there are six modes in which heat absorption occurs at the top boundary as shown in Figures $5 \mathrm{~A}$ and $\mathrm{B}$ for $\mathrm{Ra}=1.26 \times 10^{7}$. There is similarity of events in comparison to the top insulated case for $\mathrm{Ra}=1.26 \times 10^{6}$. Since the maximum temperature occurs toward the mid-section of the cavity, the inner cells grow faster than the outer cells, the growth of six modes leads to formation of two symmetric thermals with respect to the center-line. The instability produces buoyancy which lifts the two central cells thus forming tertiary modes $(t=130 \mathrm{~s})$; the rows of cells from the center toward the wall form partitioned co-rotating cells with the middle cell (tertiary mode) counter-rotating. The tertiary modes propagate toward the top of the cavity $(t=135 \mathrm{~s})$ from a bifurcation of a local double homoclinic orbit which signals transient effects. There is subsequent merging of the smaller cells (tertiary modes) along the bottom, forming a single homoclinic orbit, into single cells $(t=140 \mathrm{~s})$. The growth of the stratified layer from the top limits the penetration of the modes as shown in Figure 5B $(t=960 \mathrm{~s})$; thus the system transitions to four modes. These four modes become asymmetric $(t=1080 \mathrm{~s})$ and the growth of the asymmetry leads to merging of the inner and outer cells (secondary modes). Thus two asymmetric modes are formed at $t=1200 \mathrm{~s}$. This finding is significant as it implies that beyond the critical Rayleigh number the flow field becomes asymmetric. This implies that axi-symmetric models would be limited in their application to high Rayleigh numbers since this occurs for $\mathrm{Ra}=1.26 \times 10^{7}$. 


\section{Local Measures}

The local measures which characterize the behavior of the fluid as a function of system parameters are summarized in Figures 6A, B, and C. The effect of increasing the Rayleigh number is considered in Figures $6 \mathrm{~A}$ and $\mathrm{B}$ by showing the characteristic maximum velocity magnitude and temperature as a function of Ra contrasting the effect of insulated $\left(f_{t}=0\right)$ and top absorbing $\left(f_{t}=1\right)$ boundaries at $t=600 \mathrm{~s}$. The results in Figure 6A show that $\mathrm{V}_{\max }$ remains constant for both $f_{t}=1$ and $f_{t}=0$ for $\mathrm{Ra}<10^{5}$ indicating stability. Instability occurs for $\mathrm{Ra}>10^{5}$ for which the solution remains the same up to $10^{7}$. There is faster rise in velocity for the case $f_{t}=0$ for $10^{7}<\mathrm{Ra}<10^{9}$. This represents the range for

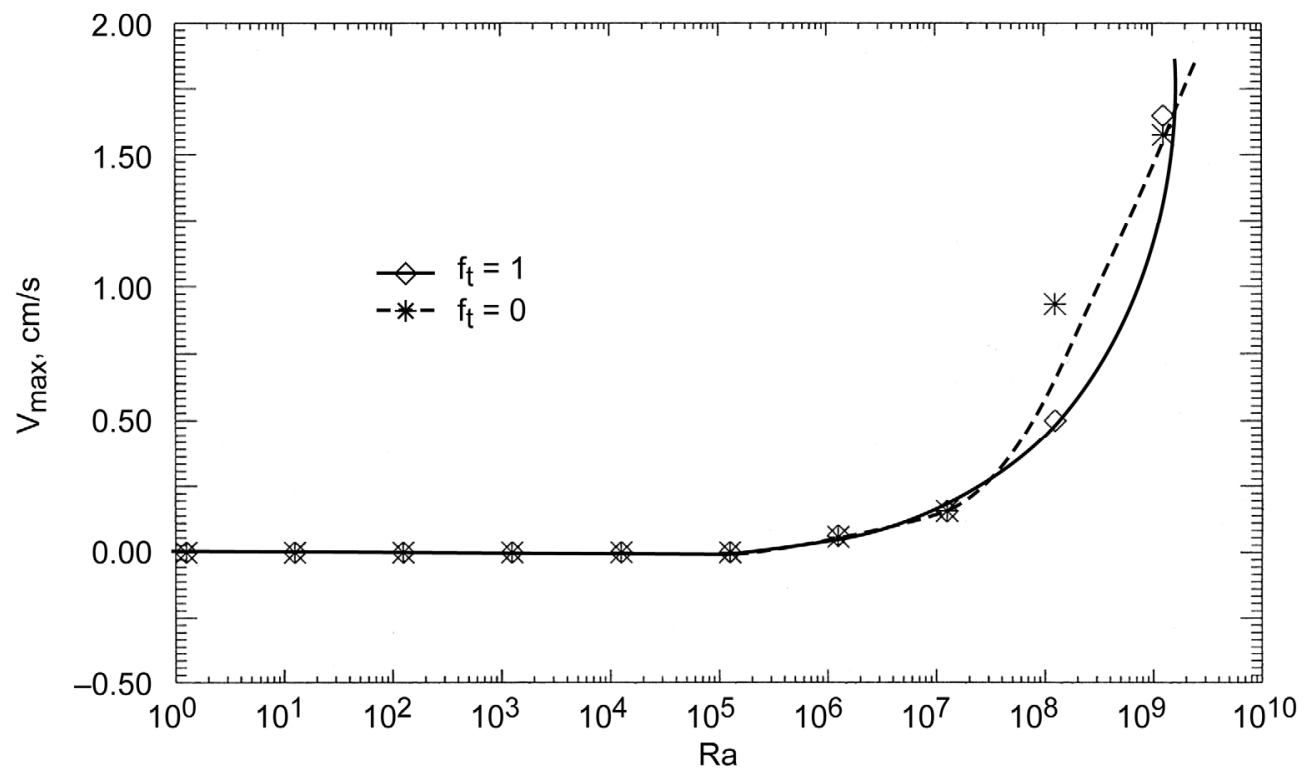

Figure 6A.-Characteristic velocity magnitude at $t=600 \mathrm{~s}$, comparing heat flux absorption $\left(\mathrm{f}_{\mathrm{t}}=1\right)$ and insulated $\left(f_{t}=0\right)$ top boundary conditions for $f_{b}=1, f_{S}=2.0 \times 10^{-6}, \operatorname{Ar}=1, \operatorname{Pr}=1.03$.

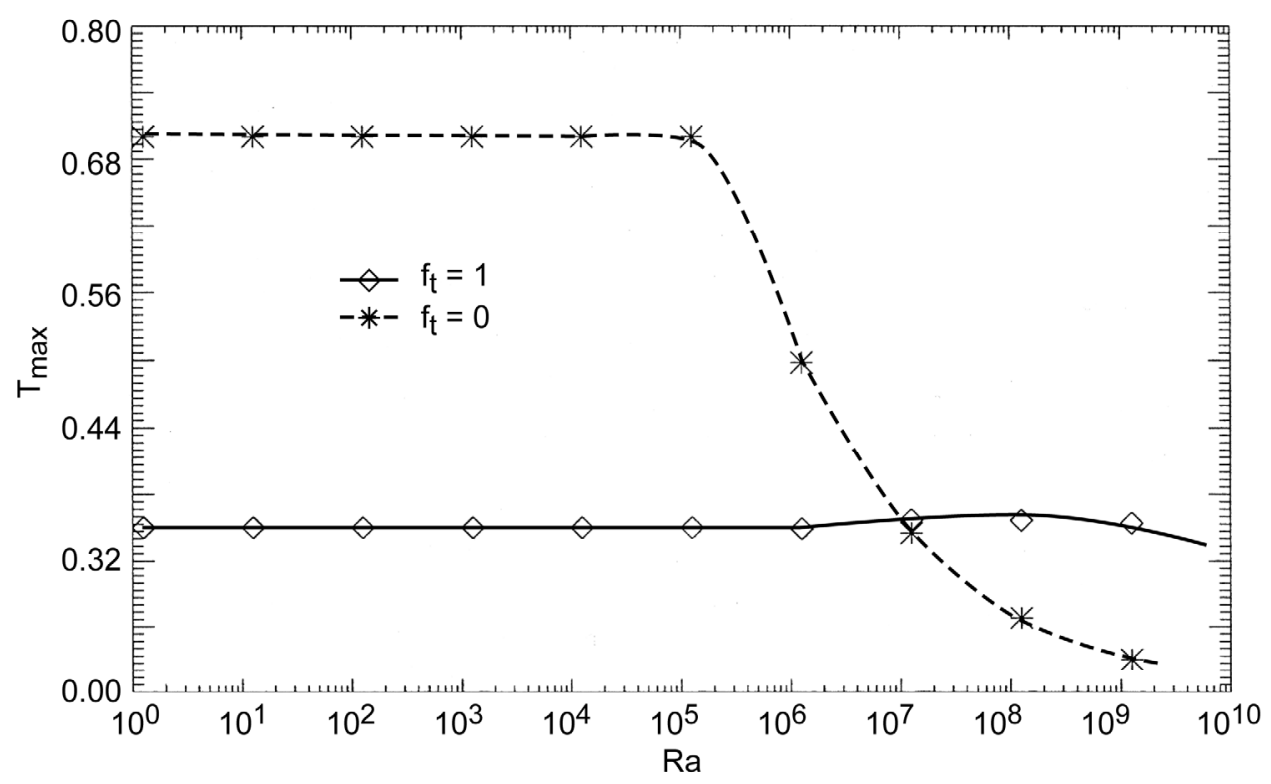

Figure 6B. - Characteristic maximum temperature at $\mathrm{t}=600 \mathrm{~s}$, comparing $\left(\mathrm{f}_{\mathrm{t}}=1\right.$ and $f_{t}=0$ ) top boundary conditions for $f_{b}=1, f_{S}=2.0 \times 10^{-6}, \operatorname{Ar}=1, \operatorname{Pr}=1.03$. 


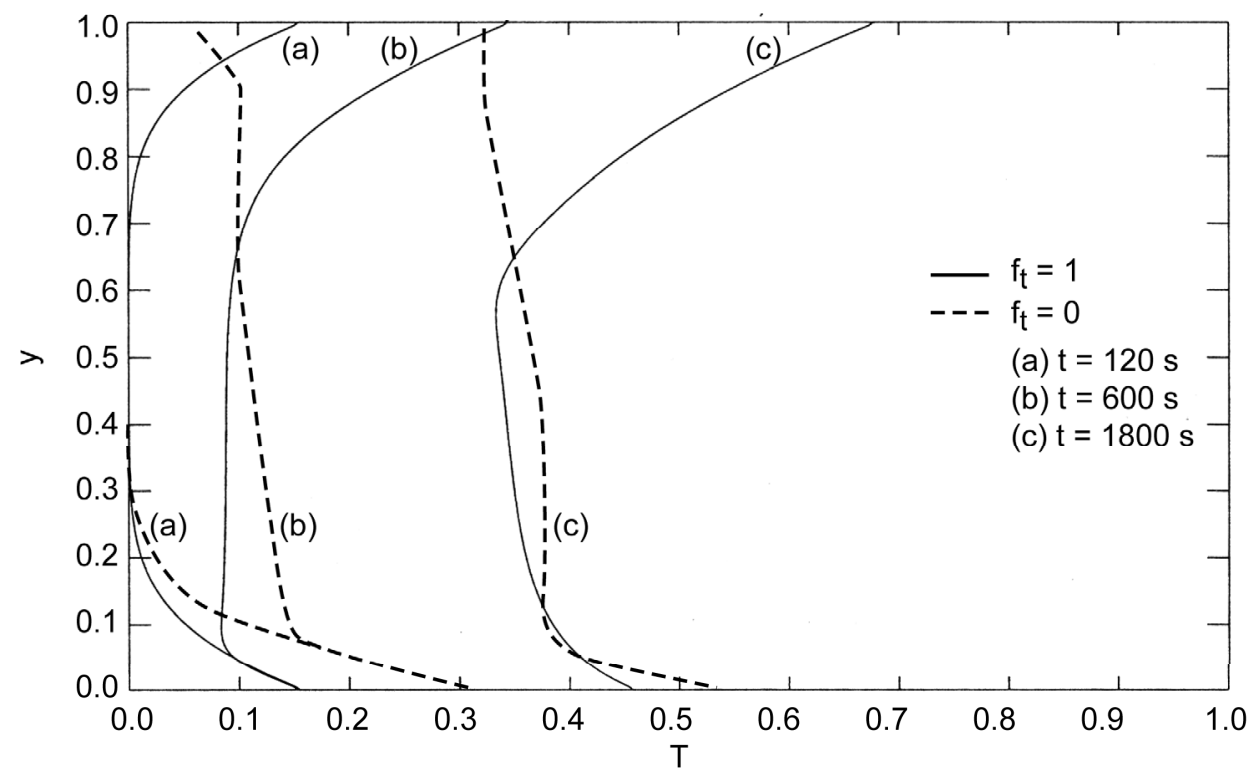

Figure 6C.-Transient response of cryogenic fluid comparing heat absorption $\left(\mathrm{f}_{\mathrm{t}}=1\right)$ to insulating $\left(f_{t}=0\right)$ top, $f_{b}=1, f_{s}=2.0 \times 10^{-6}, \operatorname{Ra}=1.26 \times 10^{6}, \operatorname{Ar}=1, \operatorname{Pr}=1.03$.

which the stabilizing effect of the top boundary $f_{t}=1$ becomes effective and lowers the convective velocity. The trend of the maximum temperature as a function of Ra in Figure $6 \mathrm{~B}$ shows that $\mathrm{T}_{\max }$ is higher for $f_{t}=0$ when $\mathrm{Ra}<10^{7}$ whereas for $\mathrm{Ra}>10^{7} \mathrm{~T}_{\max }$ becomes greater for the stabilizing top heat absorption $f_{t}=1$ case. These trends indicate that local convective mixing is much more effective at lowering $\mathrm{T}_{\max }$ for the insulated top as opposed to the case when there is heat absorption on top for $\mathrm{Ra}>10^{7}$. In terms of heat entrapment (localized increase in temperature), the trend from $f_{t}=1$ to $f_{t}=0$ is representative of expectations, that is an increase in local maximum temperature for $\mathrm{Ra}<10^{7}$ and $f_{t}<1$; since the effect of the divider is to limit heat flow, the region bounded by $0<f_{t}<1$ represents potential conditions where $\mathrm{T}_{\max }$ increases beyond the limiting condition $f_{t}=1$. The effect of the divider approximates the trend for $f_{t}<1$, thus $f_{t}=0$ represents a limiting condition for heat entrapment. For $\mathrm{Ra}>10^{7}$ the increase in convective effects for $f_{t}=0$ induces local mixing which decreases heat entrapment effects, thus a reverse trend is predicted with an asymptotic approach to a lower temperature in comparison to $f_{t}=1$. If heat entrapment is based on the condition $f_{t}<1$, indicating finite heat leakage at the divider boundary then heat entrapment is less effective for $\mathrm{Ra}<10^{7}$ when $f_{t}=1$, whereas for $\mathrm{Ra}>10^{7}$ it becomes more effective. The trend for $\mathrm{Ra}>10^{7}$ is in agreement with the trend in experimental measurements of temperature increase near the bottom divider (Ref. 1), since $\mathrm{Ra}>10^{7}$ for the experimental system. In Figure $6 \mathrm{C}$, we show the transient response of a cryogenic fluid along the central axis $(0.5, \mathrm{y})$ of the cavity for $f_{t}=1$ and $f_{t}=0$. The trend of an adverse temperature gradient at the bottom of the enclosure is not greatly affected by the top conditions. The effect of top heating $\left(f_{t}=1\right)$ is to create a stably stratified layer which increases with time. Whereas, when the top is insulated $f_{t}=0$, there is no stratified layer; the temperature becomes nearly uniform near the top as the asymptotic state is approached.

\section{Direct Numerical Simulation/Dynamical Similarity}

We demonstrated the characteristic of the flow field and the response of the temperature field for a fixed $\mathrm{Pr}, \mathrm{Ar}$, and a range of Rayleigh number for a model. We now consider the prototype used in the experiments (Ref. 1) which has much greater geometric length scales, that is $\mathrm{L}=19.1 \mathrm{~cm}, \mathrm{H}=61.9 \mathrm{~cm}$. Due to the large length scale employed in the prototype it requires a much larger grid density to resolve the small length scales in the flow and temperature fields that occurs for large Rayleigh numbers $\mathrm{Ra} \geq 10^{8}$ as shown in Figure 3B. For example the horizontal length scale of the prototype can be four times greater 
than that of the model; for a typical grid size used for the model of $200 \times 200$, the prototype would require a grid density four times as large of $800 \times 800$ to resolve the same length scale as the model. This can require more than doubling the computational resources; a typical case for a model which requires between 30 to $72 \mathrm{~h}$ using 4 Central Processing Units (CPU's) now requires between 300 to $800 \mathrm{~h}$ using 20 to 32 CPU's for the prototype. Even though, direct numerical simulation of the prototype is the most exact, dynamical similarity can be used to model the prototype. This leads to considerable savings in computational resources. We explore the application of dynamical similarity by showing that a model with smaller geometric length scale, requires matching of the parametric set $\Lambda_{P}$ (given by Eq. (22)) for the prototype to that of the model $\Lambda_{M}$; we also show that dynamical events are similar with the exception of the time scale. The issue being that due to reduction of length scale of the model, the equivalent heat flux diffusing through the model takes much shorter time. The model allows the dynamical events to be accelerated, hence the savings in computational resources.

In order to address direct numerical simulation of the prototype (for a geometric length scale $\mathrm{L}=19.1 \mathrm{~cm}, \mathrm{H}=61.9 \mathrm{~cm})$, we consider $\mathrm{Ar}=1$ as a base case, $\mathrm{Ar}=1.32(\mathrm{H} / 2)$ for the half scale model in Figure $1 \mathrm{~B}$, and $\mathrm{Ar}=3.26(\mathrm{H})$ for the full length of the prototype in Figure 1A. The external condition for the prototype is absorption of heat on the order of $1 \mathrm{~mW}$ for the top and bottom boundaries, due to ambient background laboratory condition, which is equivalent to its projection on the surface of a circle with heat flux of q" $=34.9 \mathrm{erg} / \mathrm{cm}^{2}$-s for liquid nitrogen. The condition at the sidewall is heat absorption on the order of $1 \mu \mathrm{W}$ or a heat flux q" $=0.001 \mathrm{erg} / \mathrm{cm}^{2}$-s. The characteristics of the dynamical evolution of the flow field is illustrated for $\mathrm{Ar}=1$ in Figures 7A, B, and C which show three main events: (a) transition to short wavelength instability and growth of thermals, (b) vertical propagation of the instability, and (c) a travelling wave-type mode which eventually transitions to asymmetry.

Even though the cavity size for the prototype in Figure 7A is much larger than a typical model shown in Figure $3 \mathrm{~A}\left(\mathrm{Ra}=1.26 \times 10^{9}\right)$, for nearly equivalent Rayleigh numbers and slightly different Prandtl number, similar transition instability occurs in Figure 3A at $t=12 \mathrm{~s}$ as compared to Figure 7A at $t=288 \mathrm{~s}$ for $\mathrm{Ra}=1.0 \times 10^{9}$ and $\operatorname{Pr}=2.27$. In this case there is a deceleration of the instability since the size of the cavity is so large for a given heat flux input. There is a similar growth of thermals to that shown in Figure $5 \mathrm{~A}$, however the higher grid density used $(500 \times 500)$ allows the resolution of small length scales such as the fine structures during the interaction between thermal pairs shown in Figure 7A at $t=432 \mathrm{~s}$. The propagation of the instability toward the top of the cavity occurs via a repetitive sequence of growth of secondary cells in Figure 7B. The mechanism of the instability occurs through the formation of homoclinic orbits ( $t=648 \mathrm{~s}$ and $t=720 \mathrm{~s})$, which serve to increase the size of the cells and form secondary cells inside the main cell, which subsequently detaches and form secondary cells as shown from the transition between $t=720 \mathrm{~s}$ to $t=792 \mathrm{~s}$. Once the flow field penetrates near the top of the cavity, there is a travelling wave-type mode event that occurs as shown in Figure 7C. The travelling wave-type mode occurs through the exchange of energy between tertiary and secondary modes as shown for $t=864 \mathrm{~s}, t=1296 \mathrm{~s}$, and $t=1368 \mathrm{~s}$. As the travelling wave-type mode propagates up and down the cavity there is a breakdown and reconnection of the vertical modes; there is also boundary layer entrainment along the vertical walls. The flow field subsequently transitions to asymmetry. Note in comparison to Figures 5A and B the boundary layer extends further up the cavity vertical walls.

\section{Effect of Aspect Ratio}

The effect of increasing the height of the cavity to actual prototype dimension model $\mathrm{Ar}=1.62(\mathrm{H} / 2)$ and $\mathrm{Ar}=3.24(\mathrm{H})$ for long and short time is shown in Figure 8 contrasting insulated $f_{t}=0$ and absorbing top $f_{t}=1$ boundary conditions. For the case of a taller cavity $\mathrm{Ar}=1.62$, the growth of tertiary modes is formed between two co-rotating secondary cells. The tertiary mode has a homoclinic orbit which signals propagation of a travelling wave inside a fluid. This case is contrasted with $\mathrm{Ar}=3.72$, the prototype used in the experiments (Ref. 1). Such a large cavity requires high grid density to resolve small scales; we show direct numerical simulation of early time evolution of the instability using $1000 \times 1000$ grids for 
short times. The results show the onset of instability which consists of six modes segregated toward the wall with small length scales. In this case, the characteristic time for the instability to occur increases $(t=288 \mathrm{~s})$ due to the large increase in vertical length scale as compared to a model in Figure 3A which shows the instability at $t=12 \mathrm{~s}$; note the instability develops faster with a lower aspect ratio prototype as in Figure 7A at $t=288 \mathrm{~s}$.
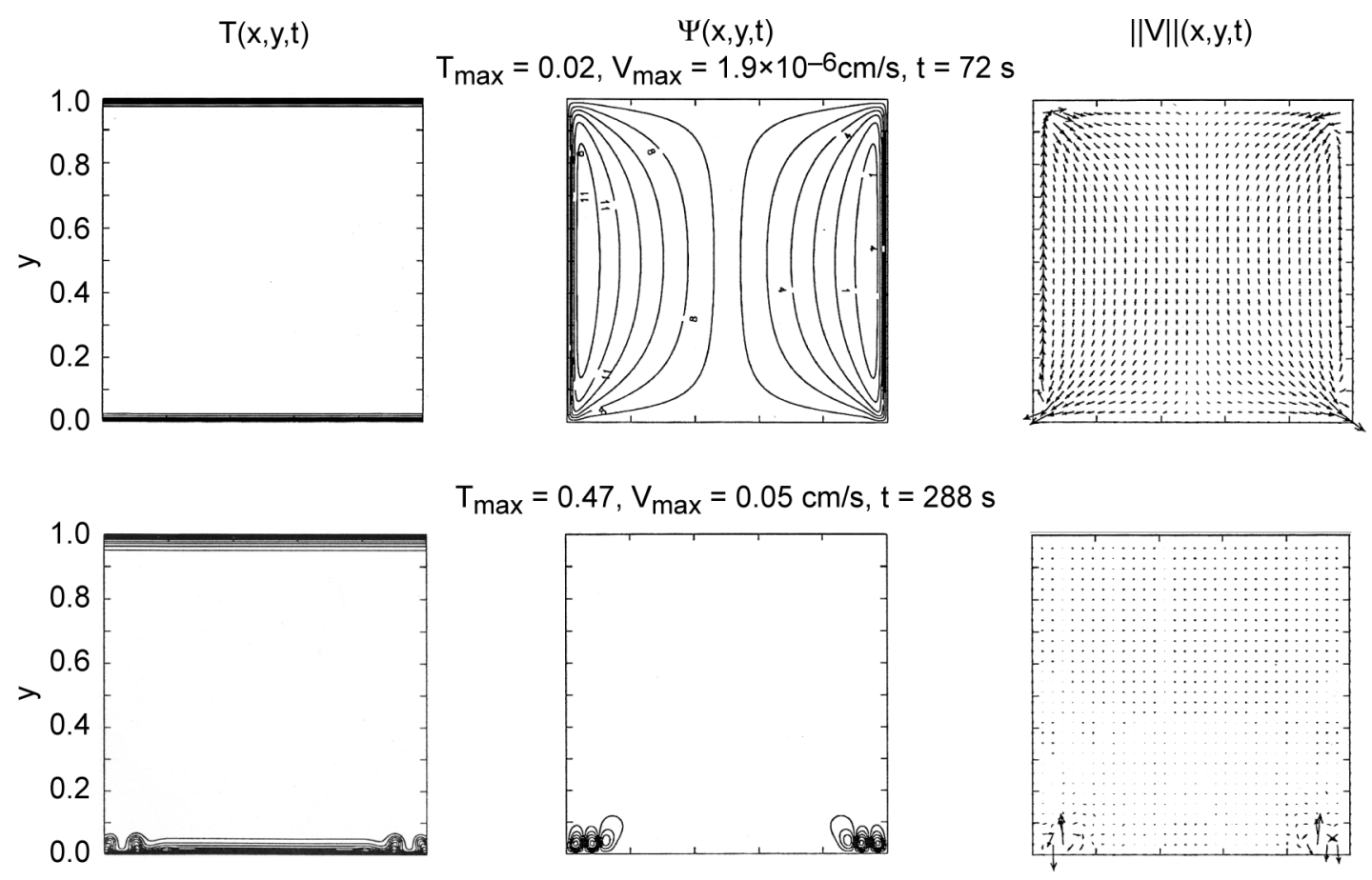

$\mathrm{T}_{\max }=0.47, \mathrm{~V}_{\max }=0.05 \mathrm{~cm} / \mathrm{s}, \mathrm{t}=288 \mathrm{~s}$
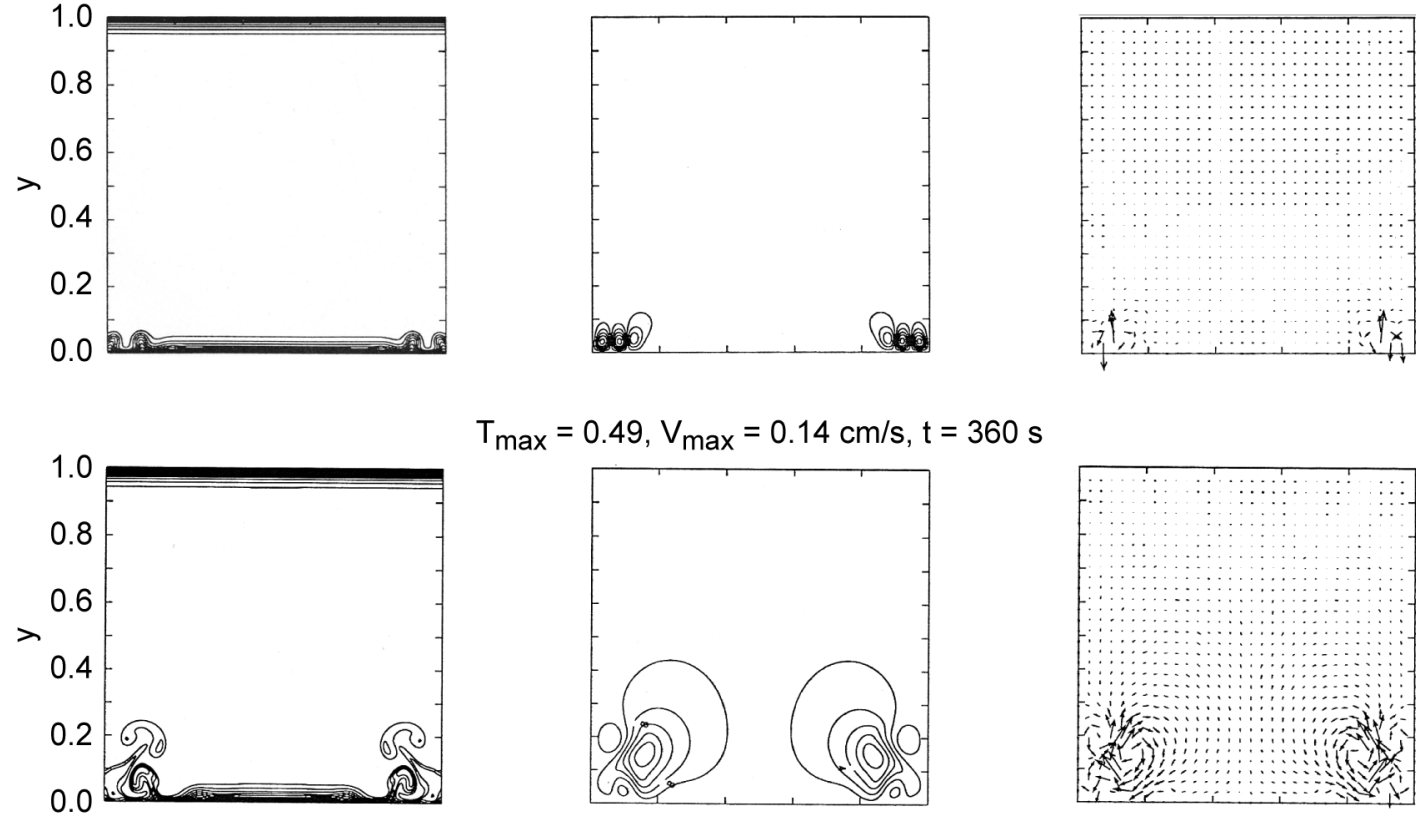

$\mathrm{T}_{\max }=0.49, \mathrm{~V}_{\max }=0.14 \mathrm{~cm} / \mathrm{s}, \mathrm{t}=360 \mathrm{~s}$
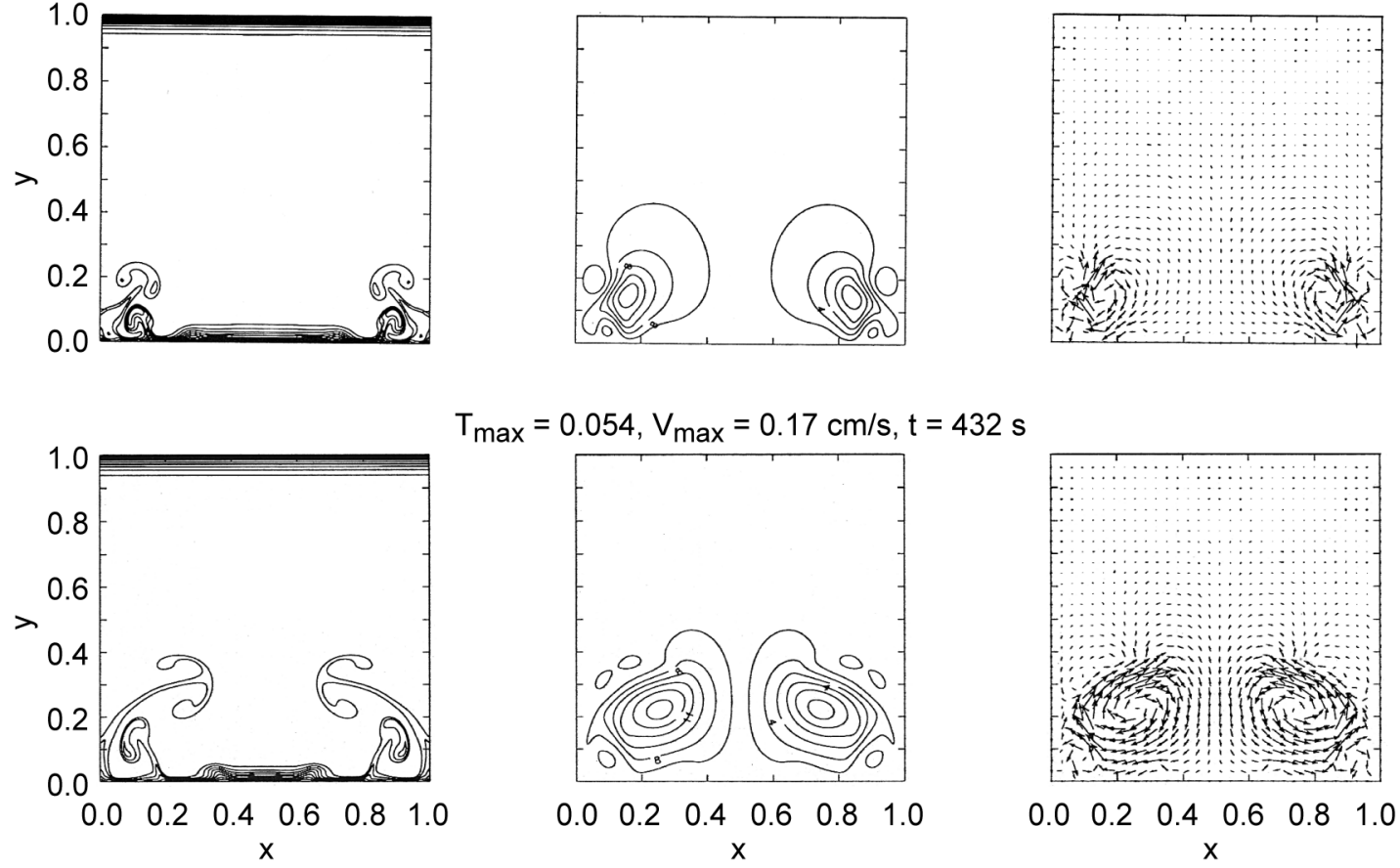

Figure 7A.-Transition from short wavelength instability to nonlinear growth of thermals $\left(f_{b}=1, f_{t}=1\right.$, $\left.f_{S}=2.9 \times 10^{-5}\right)$ for prototype $(L=H=19.1 \mathrm{~cm}) ; q^{\prime \prime}=34.9 \mathrm{erg} / \mathrm{cm}^{2}-\mathrm{s}, \mathrm{Ra}=1.0 \times 10^{9}, \mathrm{Ar}=1, \operatorname{Pr}=2.27$ (grid size $500 \times 500$ ). 


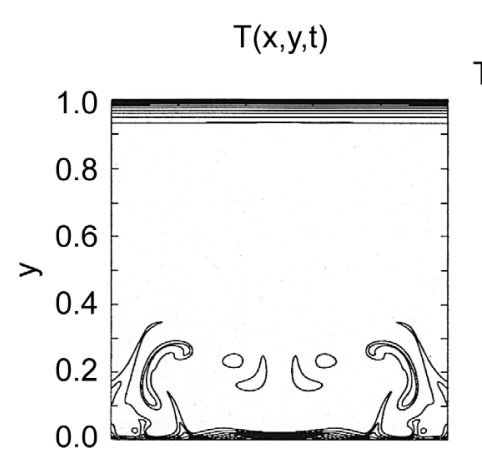

$\Psi(\mathrm{x}, \mathrm{y}, \mathrm{t})$

$\mathrm{T}_{\max }=0.058, \mathrm{~V}_{\max }=0.16 \mathrm{~cm} / \mathrm{s}, \mathrm{t}=504 \mathrm{~s}$
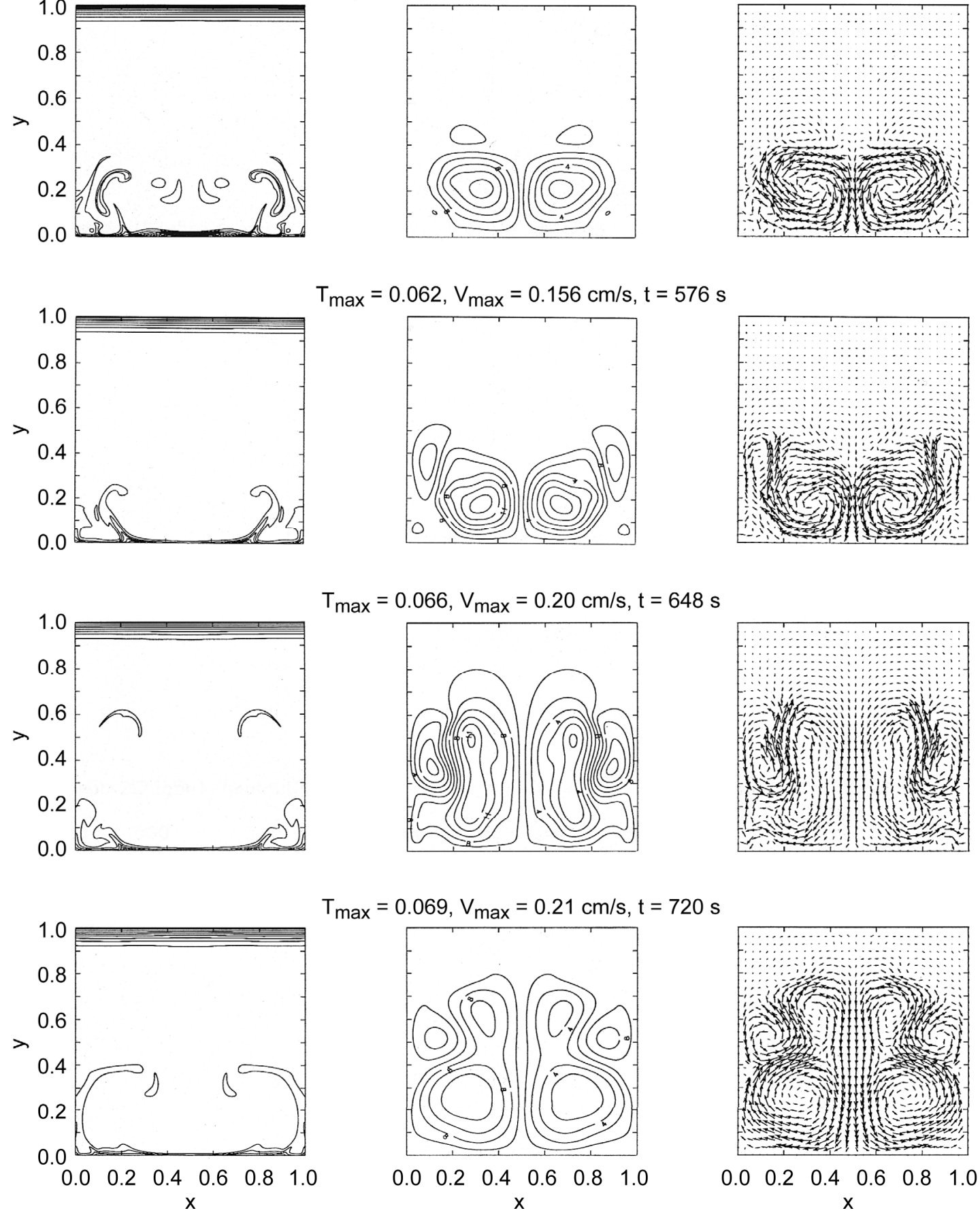

Figure 7B.-Propagation of instability, $\mathrm{Ra}=1.0 \times 10^{9}, \mathrm{Ar}=1, \mathrm{Pr}=2.27$. 

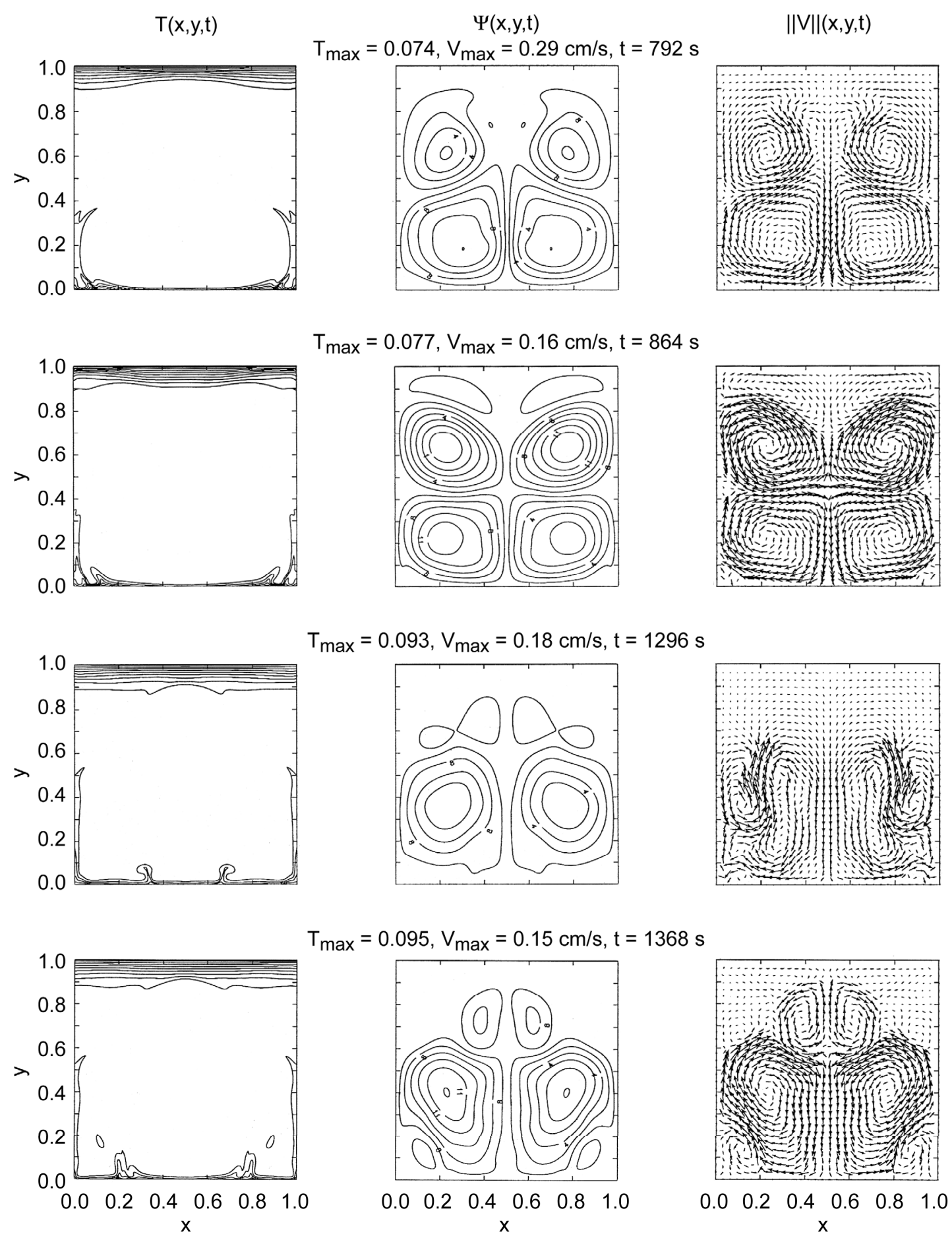

Figure 7C.-Travelling wave-type motion of convective modes, $\operatorname{Ra}=1.0 \times 10^{9}, \operatorname{Ar}=1, \operatorname{Pr}=2.27$. 


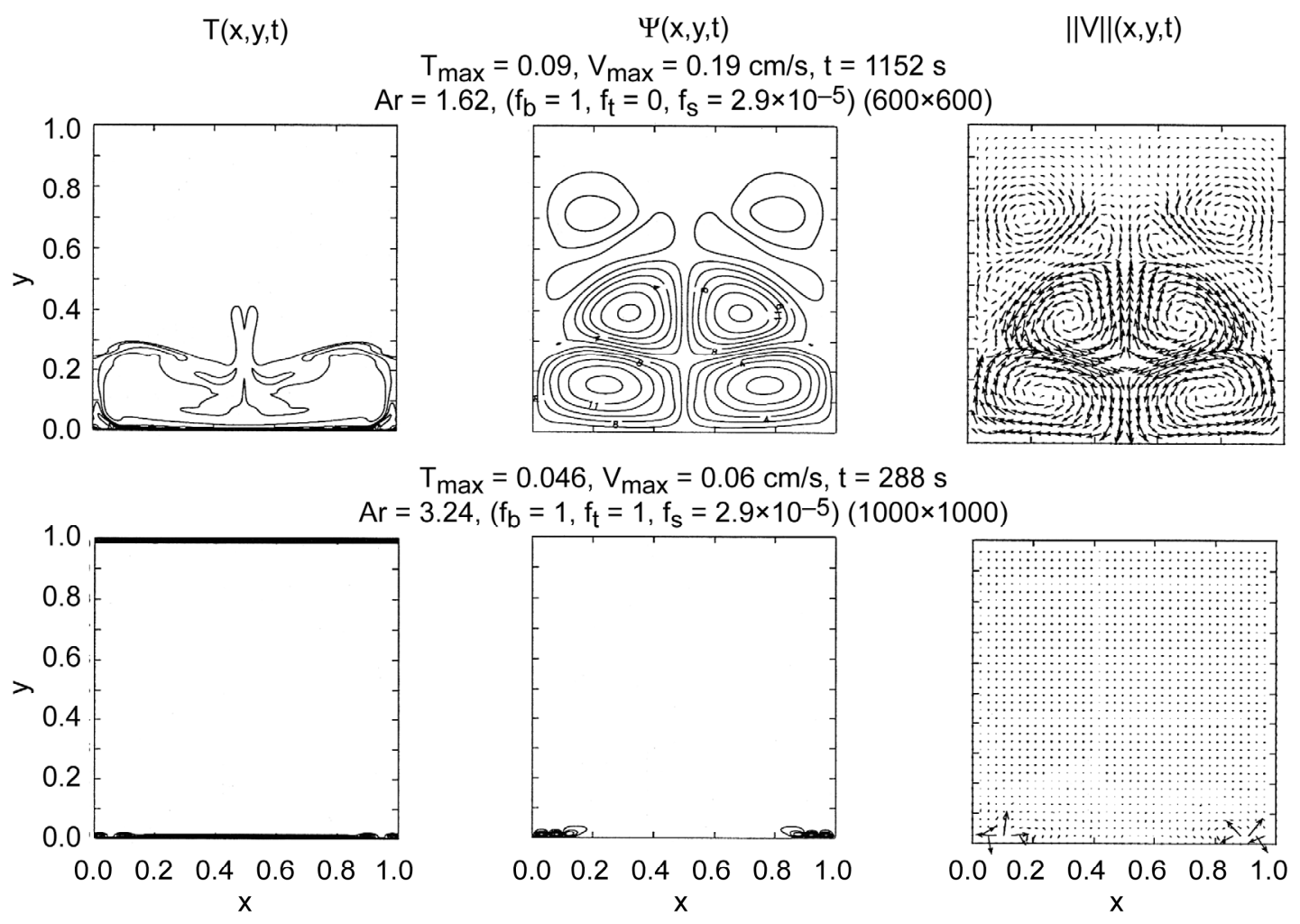

Figure 8.-Effect of aspect ratio on propagation of instability, $(\mathrm{Ar}=1.62, \mathrm{H}=30.95 \mathrm{~cm})$ and onset of short wavelength instability $(\mathrm{Ar}=3.24, \mathrm{H}=61.9 \mathrm{~cm})$ for prototype $(\mathrm{L}=19.1 \mathrm{~cm}), \mathrm{q}^{\prime \prime}=34.9 \mathrm{erg} / \mathrm{cm}^{2}-\mathrm{s}$, $\operatorname{Pr}=2.27, \mathrm{Ra}=1.0 \times 10^{9}$.

\section{Application of Dynamical Similarity}

We illustrate the application of dynamical similarity using a model in Figure 9 with smaller length scale for the same heat flux boundary condition as the prototype in Figure 7A. In this case,

$$
\Lambda_{M}\left(R a, A r, \operatorname{Pr}, f_{s}, f_{b}, f_{t}\right)=\Lambda_{P}\left(R a, A r, \operatorname{Pr}, f_{s}, f_{b}, f_{t}\right)
$$

note the matching of the parametric space yields similar dynamical events as compared to Figures 7A, B, $\mathrm{C}$ except with the model the characteristic time for the instability to develop is much shorter $t=20 \mathrm{~s}$ as compared to $t=288 \mathrm{~s}$ for the prototype in Figure 7A.

\section{Local Flow Dynamics at Fixed Points}

The local dynamics of the flow and temperature fields at fixed points $\left(\mathrm{x}_{\mathrm{p}}, \mathrm{y}_{\mathrm{p}}\right)$ as denoted in Figure 1A(b) is shown in Figure 10. The decomposition of the time history signal into its frequency components for the flow field is obtained by estimating its power spectrum using,

$$
\tilde{P}_{V}(f)=\frac{1}{T_{b}}\left|\int_{0}^{T_{b}} V(t) e^{-2 \pi i f t}\right|^{2}
$$

in which $\mathrm{V}(\mathrm{t})$ is the time history of the corresponding component of the velocity field at the time interval of the data denoted by $\mathrm{T}_{\mathrm{b}}$. The smooth estimate $\mathrm{P}_{\mathrm{V}}(\mathrm{t})$ is obtained from a convolution relationship $\left(P_{V}(f)=W_{H}(f) * \tilde{P}_{V}(f)\right)$ using a Hanning spectral window $\left(\mathrm{W}_{\mathrm{H}}(\mathrm{f})\right)$. The dynamical characteristics of 

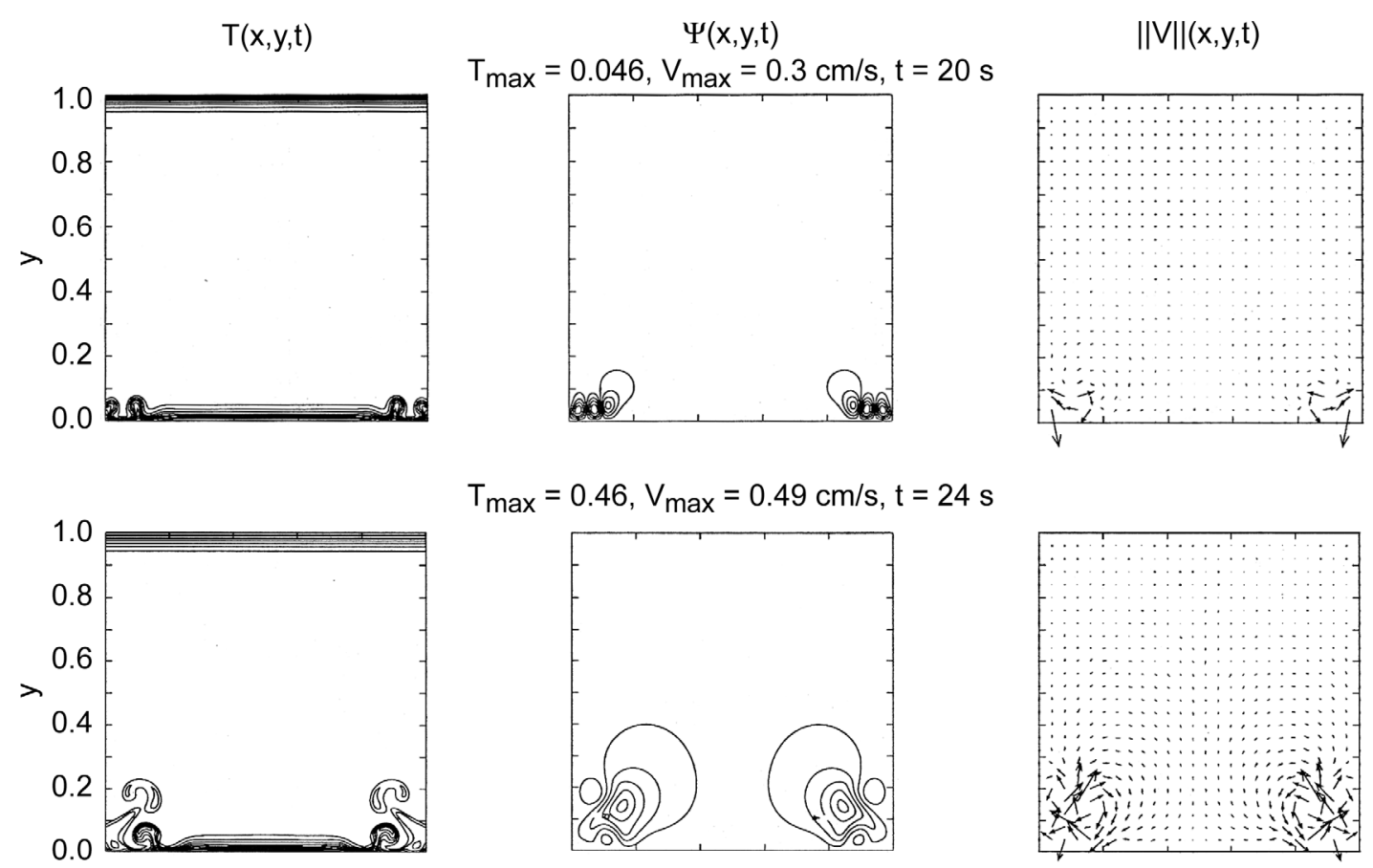

$\mathrm{T}_{\max }=0.46, \mathrm{~V}_{\max }=0.49 \mathrm{~cm} / \mathrm{s}, \mathrm{t}=24 \mathrm{~s}$
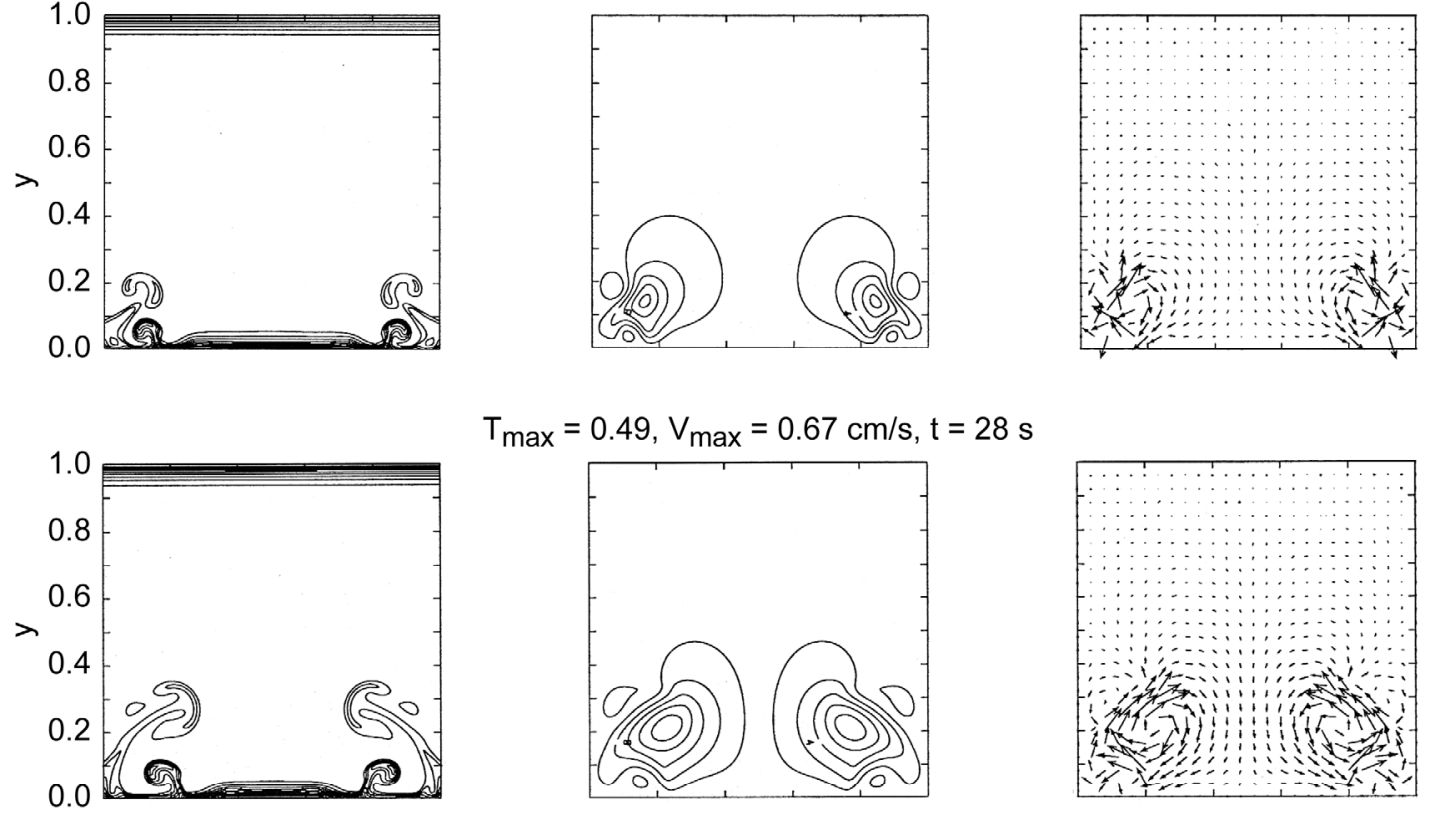

$\mathrm{T}_{\max }=0.49, \mathrm{~V}_{\max }=0.67 \mathrm{~cm} / \mathrm{s}, \mathrm{t}=28 \mathrm{~s}$
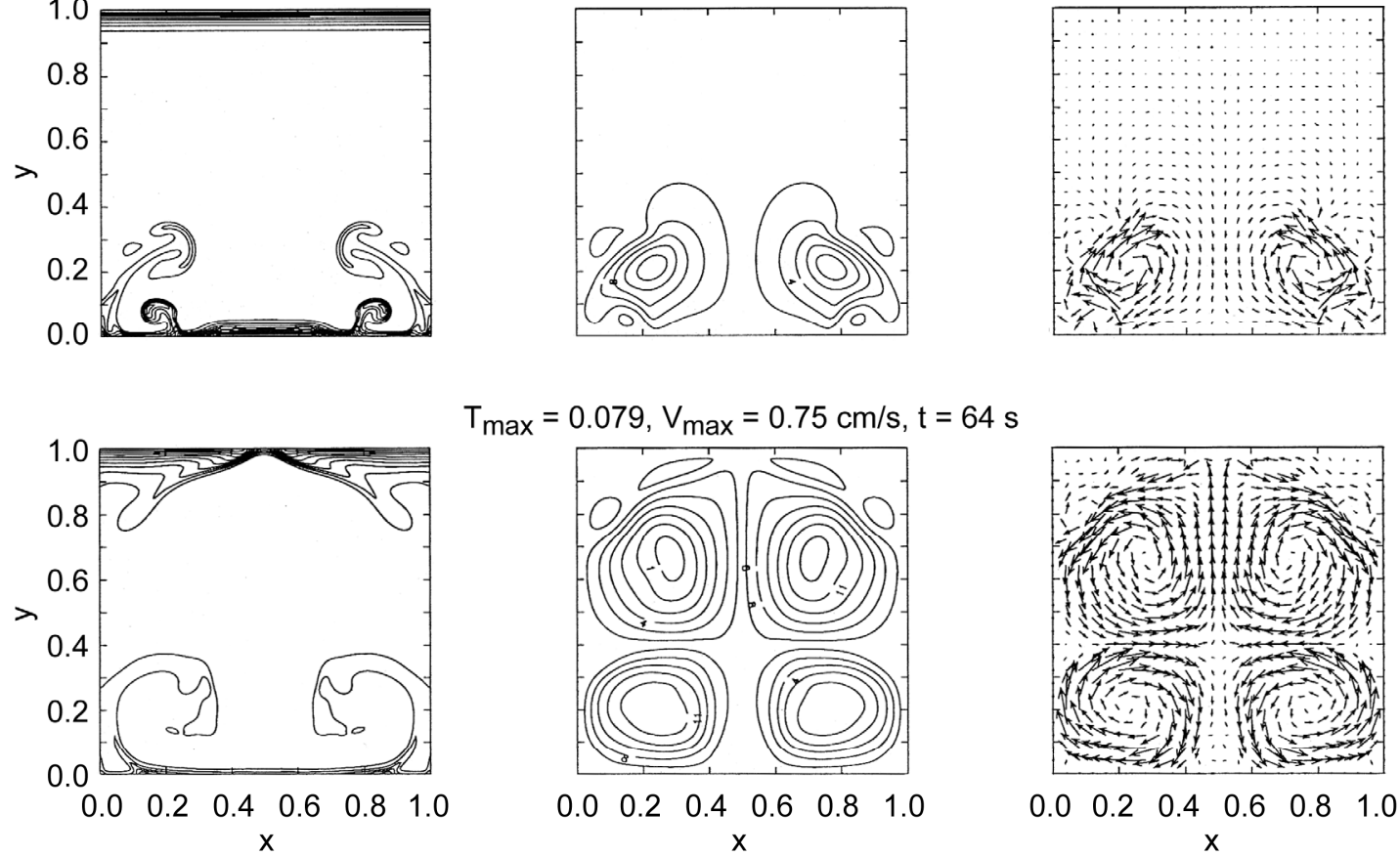

Figure 9.-Similarity in dynamical events for model $(\mathrm{L}=\mathrm{H}=5 \mathrm{~cm}, \mathrm{Ar}=1)$ with smaller cavity size $\left(f_{b}=1, f_{t}=1, f_{s}=2.9 \times 10^{-5}\right), \operatorname{Pr}=2.27, q^{\prime \prime}=34.9 \mathrm{erg} / \mathrm{cm}^{2}-\mathrm{s}, \mathrm{Ra}=1.0 \times 10^{9}$ (grid size $\left.200 \times 200\right)$. 

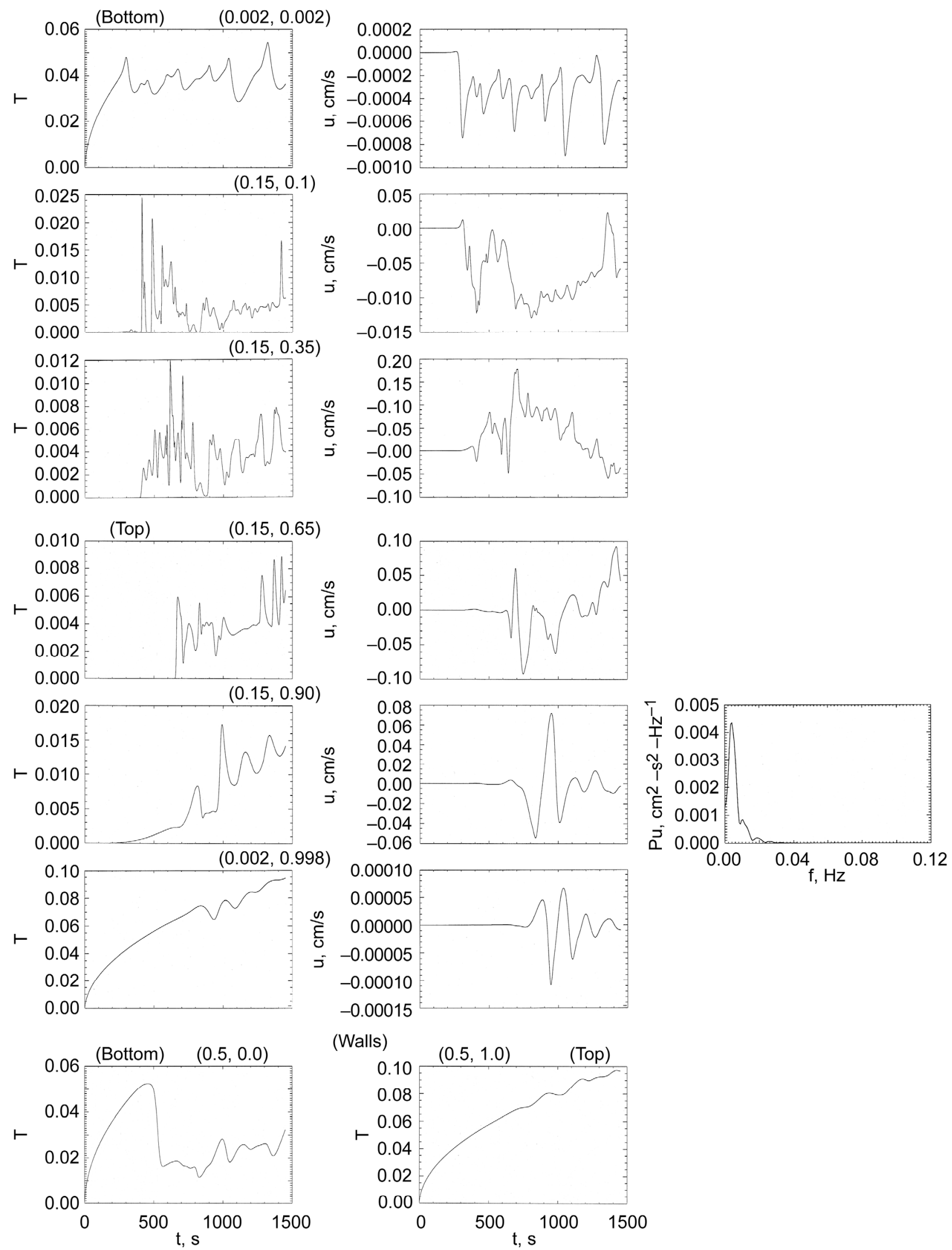

(Walls)

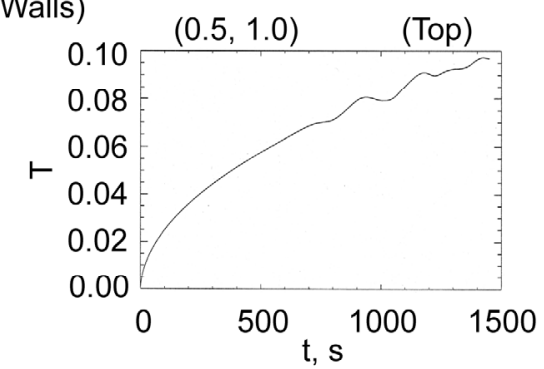

Figure 10.-Dynamical characteristics of flow and temperature fields at various fixed points $\left(x_{p}, y_{p}\right)$ for prototype, $\operatorname{Ar}=1, \operatorname{Pr}=2.27, \operatorname{Ra}=1.0 \times 10^{9}($ grid size $500 \times 500)$. 
flow and temperature fields at various fixed points $\left(\mathrm{x}_{\mathrm{p}}, \mathrm{yp}\right)$ for the prototype are shown in Figure 10. In relation to Figure $1 \mathrm{~A}(\mathrm{~b})$, the series of points represent location near the left wall which proceeds from the bottom toward the top for a time interval $T_{b}=1500 \mathrm{~s}$. As shown in Figure 10, the time history of the temperature and flow fields in the bottom region at $(0.002,0.002)$ shows that for a typical q" $=$ $34.9 \mathrm{erg} / \mathrm{cm}^{2}-\mathrm{s}$ in which $\left(f_{b}=1, f_{t}=1, f_{s}=2.9 \times 10^{-5}\right)$, the temperature increases initially by conduction effects up to $t=300 \mathrm{~s}$ while the flow field remains quiescent; for $t>300 \mathrm{~s}$ the onset of instability occurs in which the flow and temperature fields oscillate aperiodically in the core region. As the distance increase from $(0.15,0.1)$ to $(0.15,0.35)$ at the bottom, it takes longer $(t=400 \mathrm{~s})$ for the temperature and flow fields to respond and there is also an increase in their aperiodicity. As the distance is further increased toward the top of the cavity, from $(0.15,0.65)$ to $(0.002,0.998)$, the flow and temperature oscillations transition from aperiodic to a nearly periodic (mono-frequency) oscillation. This trend is due to absorption of heat $\left(f_{t}=1\right)$ at the top boundary which stabilizes the flow. The power spectrum shows that there is a dominant mono-frequency component of $0.004 \mathrm{~Hz}$ with slight harmonics at $(0.15,0.90)$; this corresponds to a long period of $250 \mathrm{~s}$. Note that near the top $(0.002,0.998)$ conduction effects dominate and it takes on the order of $t=800 \mathrm{~s}$ for the onset of convective instability. The behavior of the wall temperature at the mid-section of the bottom $(0.5,0.0)$ and top $(0.5,1.0)$ shows that at the bottom convective instability $(t>400 \mathrm{~s})$ results in a decrease in temperature due to cooling by the flow field whereas at the top the temperature increases beyond the onset of oscillations $(t>800 \mathrm{~s})$. These trends are representative of the behavior of the flow and temperature fields for the prototype for $\mathrm{Ra}=1.0 \times 10^{9}$.

We now consider the behavior of the flow and temperature fields for a model near the threshold of instability at $\mathrm{Ra}=1.26 \times 10^{6}$ as well as a case for $\mathrm{Ra}=1.0 \times 10^{9}$ in order to compare with the prototype. The application of dynamical similarity $\left(\Lambda_{M}=\Lambda_{P}\right)$ using a model with smaller geometric length scale, showing time asymptotic behavior near the top of the cavity at $(0.15,0.90)$, is shown in Figure 11 . Since the cavity size of the model is smaller, the equivalent amount of heat input to the bottom diffuses much faster as compared to the prototype, therefore the instability occurs much sooner. Near the threshold of instability at $\mathrm{Ra}=1.26 \times 10^{6}$ there is oscillation in both the temperature and flow fields; the power spectrum of the flow field shows oscillations with frequency of $f=0.001 \mathrm{~Hz}$ and harmonics of the frequency, $2 f, 3 f, 4 f$ $(4 f=0.004 \mathrm{~Hz})$ which spans a range of period from 250 to $1000 \mathrm{~s}$. Comparison of the model, showing a time asymptotic state, and prototype in Figure 10 at location $(0.15,0.9)$ shows that the model exhibits aperiodic behavior as time increases for $\mathrm{Ra}=1.0 \times 10^{9}$. The time asymptotic behavior of the velocity component shows intermittent periodic bursts which contain a broadband frequency content extending to $0.4 \mathrm{~Hz}$ as indicated by the power spectrum $\mathrm{P}_{\mathrm{u}}$; this dynamical state indicates a transition to chaos. We compare a sub-interval of the time history to the prototype in Figure 10 at $(0.15,0.90)$ and the remarkable results show similar behavior for both the temperature and flow fields. The initial response time of the model occurs near $t=10 \mathrm{~s}$ whereas the prototype corresponds to $t=200 \mathrm{~s}$, and the onset of convective instability occurs near $t=60 \mathrm{~s}$ whereas the prototype indicates $t=700 \mathrm{~s}$. Similar to the prototype the power spectrum indicates periodic frequency response, however the frequency $(0.04 \mathrm{~Hz})$ is ten times larger with a corresponding shorter period of $25 \mathrm{~s}$, and higher harmonics indicating nonlinear effects. Such dynamical similar results indicate that a model can be used to infer the dynamical characteristics of a prototype approximately. This finding is practical as it represents an alternate means to study the behavior of a prototype which requires significantly less computational resources; for example a typical prototype that requires $800 \mathrm{~h}$ to run may only require $40 \mathrm{~h}$ for a model. The major difference between the model and prototype is the characteristic time for the onset of instability, the model requires much shorter time, hence the savings in computational time. 


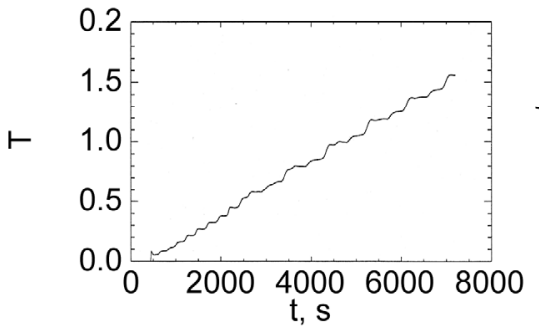

$\mathrm{Ra}=1.26 \times 10^{6}, \mathrm{Pr}=1.03,(0.15,0.9)$

$\left(\mathrm{f}_{\mathrm{b}}=1, \mathrm{f}_{\mathrm{t}}=0, \mathrm{f}_{\mathrm{s}}=2 \times 10^{-6}\right)$
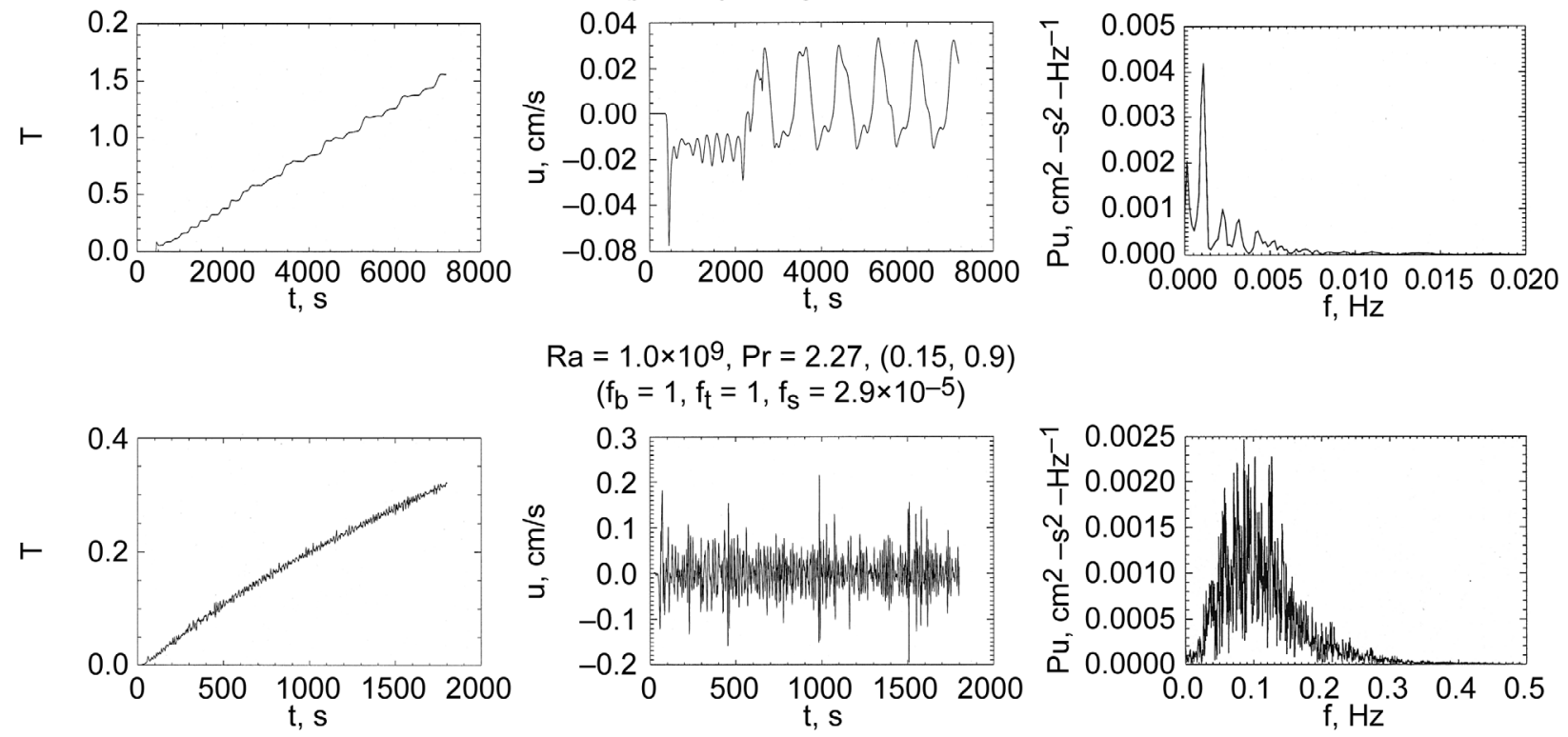

$\mathrm{Ra}=1.0 \times 10^{9}, \mathrm{Pr}=2.27,(0.15,0.9)$

$\left(f_{b}=1, f_{t}=1, f_{s}=2.9 \times 10^{-5}\right)$
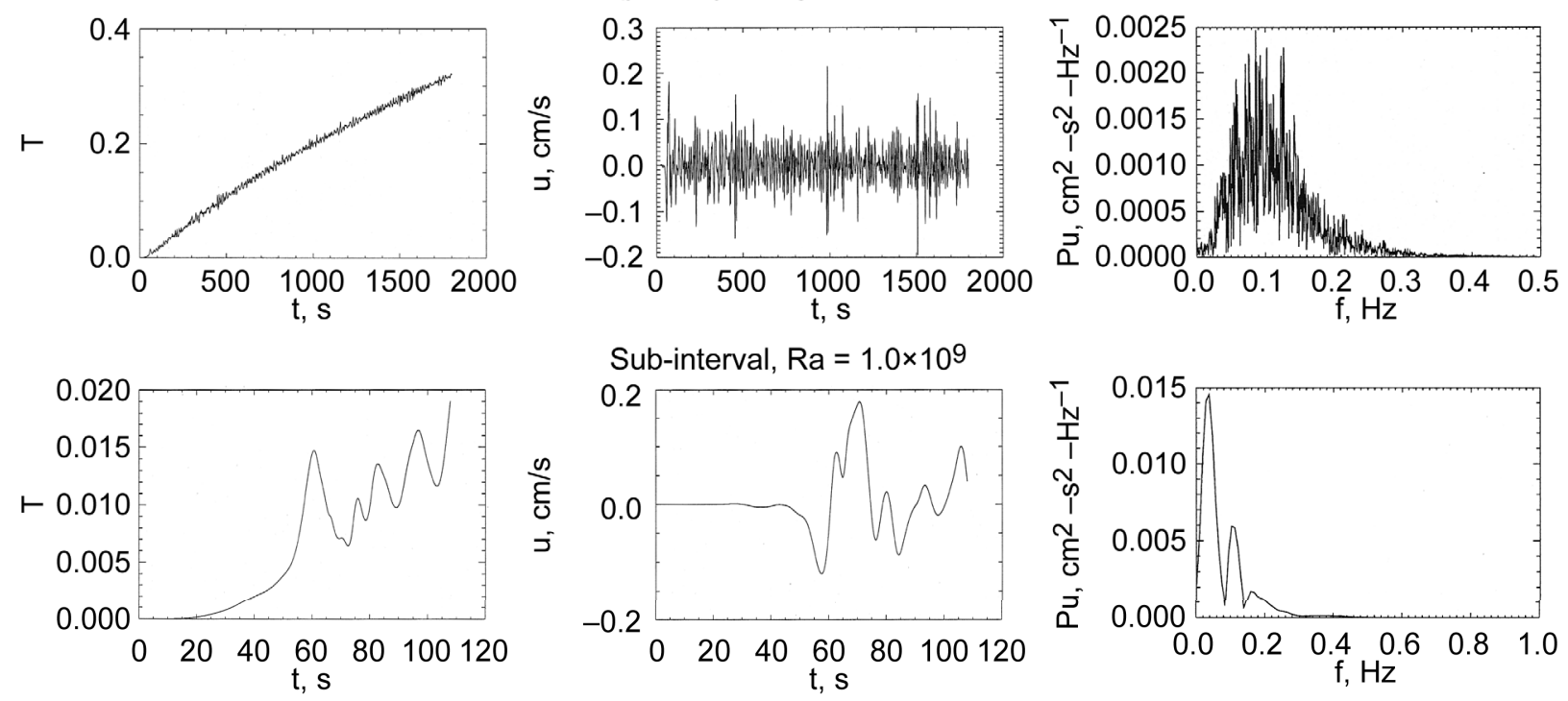

Figure 11.-Similarity in dynamical characteristics for model $(\mathrm{Ar}=1, \mathrm{~L}=\mathrm{H}=5 \mathrm{~cm})$ at various Rayleigh numbers, (grid size $200 \times 200$ ).

The effect of aspect ratio on the prototype for $\mathrm{Ar}=1.6$ and $\mathrm{Ar}=3.24$ is contrasted for long and short times in Figure 12. For $\mathrm{Ar}=1.6$, we show a case for $\mathrm{Ra}=1.0 \times 10^{9}$ corresponding to subcooling at the top boundary $\left(f_{t}=-0.1\right)$ which is relatively low. The time history of the temperature and flow fields near the bottom of the enclosure at $(0.0025,0.0017)$ is similar to that in Figure 10 shown at $(0.002,0.002)$. Thus low subcooling on the top boundary does not have a significant effect on the dynamical characteristics of the flow field. We show a full scale simulation of the prototype for $\mathrm{Ar}=3.24$ in which the grid density is increased from $400 \times 600(\mathrm{Ar}=1.6)$ to $1000 \times 1000$; this is demonstrated for the baseline condition in which the same amount of heat flux is absorbed at both the bottom and top boundary $\left(f_{b}=1, f_{t}=1\right)$ for short times. The results show that it takes on the order of $300 \mathrm{~s}$ for the onset of instability; this corresponds to approximately $800 \mathrm{~h}$ of computational time. To compute the time asymptotic behavior requires significant resources to reach the asymptotic state. The wall temperature at the bottom $(0.5,0.0)$ shows a monotonic increase without fluctuations; this occurs because for the short time considered conduction effects are dominant. In comparison to the parametric range of the experiment (Ref. 1), the upper limit of imposed heat flow at the bottom boundary is $104 \mathrm{~W}$ which corresponds to approximately $\mathrm{q}_{\mathrm{b}} "=3.49 \times 10^{6} \mathrm{erg} / \mathrm{cm}^{2}-\mathrm{s}$. In order to find the baseline condition to estimate the heat flux absorbed from the top and bottom boundary we assumed a heat flow of approximately $1 \mathrm{~mW}$, from background environment, which corresponds to a heat flux of $\mathrm{q}_{\mathrm{b}} "=3.49 \times 10^{1} \mathrm{erg} / \mathrm{cm}^{2}$-s. The scaling of the temperature magnitude using Equation (13), for comparison with experimental measurements (Ref. 1) for the baseline condition of no screen, indicates that the heat flux absorbed at the boundary is greater than the assumed value of $\mathrm{q}_{\mathrm{b}}{ }^{\prime}=3.49 \times 10^{1} \mathrm{erg} / \mathrm{cm}^{2}-\mathrm{s}$ and lies in the range of $3.49 \times 10^{1} \mathrm{erg} / \mathrm{cm}^{2}-\mathrm{s}<\mathrm{q}_{\mathrm{b}}{ }^{\prime}<3.49 \times 10^{6} \mathrm{erg} / \mathrm{cm}^{2}-\mathrm{s}$. Using a combination of dynamical 

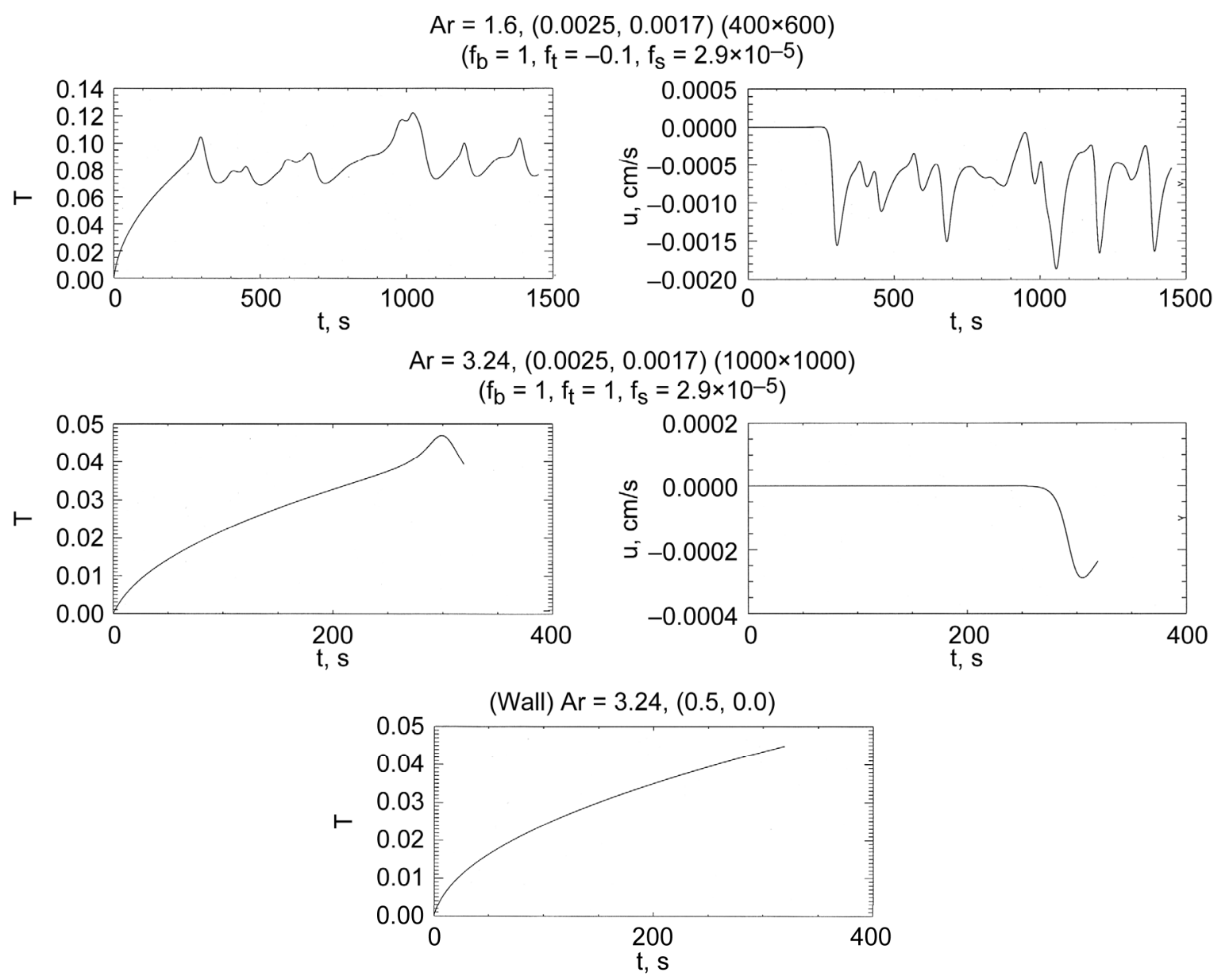

Figure 12.-Effect of aspect ratio on dynamical characteristics of prototype with $(\mathrm{Ar}=1.6, \mathrm{~L}=19.1 \mathrm{~cm}$, $\mathrm{H}=30.95 \mathrm{~cm})$ and $(\mathrm{Ar}=3.24, \mathrm{~L}=19.1 \mathrm{~cm}, \mathrm{H}=61.9 \mathrm{~cm})$ for $\operatorname{Pr}=2.27, \mathrm{Ra}=1.0 \times 10^{9}$.

similarity and full scale simulation of the prototype, it remains to investigate the effect of higher heat flux absorption at the boundaries. However the parametric range considered, for low heat flux condition at the top and bottom boundaries, provides a foundation to investigate heat entrapment effects at higher Rayleigh numbers.

\section{Summary and Conclusions}

We address heat entrapment effects with application to liquid acquisition device (LAD) inside cryogenic storage tanks. We use dynamical similarity for the model along with direct numerical simulation of the prototype with much larger geometric length scale to address the global dynamics of the flow and temperature fields and local dynamics at fixed points. From scaling analysis, we show that the parametric space depends on six parameters namely ( $\mathrm{Ra}, \operatorname{Pr}, \mathrm{Ar}, f_{s}, f_{b}, f_{t}$ ) the Rayleigh and Prandtl numbers, the aspect ratio, and the corresponding heat flux ratios for the side, bottom, and top boundaries. Due to the large parametric set involved, we show a selected parametric range based on typical experimental conditions applicable to LAD. The parametric set entails Ra between 1 and $1.26 \times 10^{9}, \mathrm{Ar}$ between 1 and 3.24, $\operatorname{Pr}=1.03$ and 2.27, $\left(f_{s}, f_{b}\right)$ between 0 and 1 , and $f_{t}$ between -0.1 to 1 . We address particularly the effect of applied heat flux at the boundaries to establish a baseline condition for a nonequilibrium cryogenic fluid inside an enclosure absorbing heat due to background laboratory environment. This serves to establish the conditions likely to occur at the top and side boundaries which can be used for simulation with known input heat flux to the bottom boundary. 
The results show that for typical conditions, convection is driven via the interaction between natural convection driven by heat flux absorbed along the vertical walls and convective instability due to heat flux absorbed along the horizontal bottom boundary. Heat flux absorbed at the top boundary serves as a stabilizing mechanism. The predominant mechanism for convection is convective instability for which the longest wavelength occurs for Ra on the order of $10^{5}$ which is near the threshold of instability. The maximum number of modes that occur is six for Ra of order $10^{7}$; these modes segregate toward the wall as $\mathrm{Ra}$ increases to $1.26 \times 10^{9}$. The wavelength of the horizontal modes decreases as Ra increases which governs the length scale that needs to be resolved for computations of high Rayleigh numbers. For Rayleigh numbers near the threshold of instability the flow field remains symmetric, however for Ra on the order $10^{7}$ there is a transition to asymmetric flow.

Direct numerical simulation for large geometric length scale prototype for $\mathrm{Ra}=1.0 \times 10^{9}$ shows that the global dynamics of the flow field evolve in three stages. These stages consist of a transition from short wavelength instability to nonlinear growth of thermals, propagation of the flow field to the top of the cavity via a repetitive sequence of destabilization which gives rise to secondary and tertiary modes, and a travelling wave-type motion of convective modes which occur prior to transition to asymmetry. The effect of increasing the aspect ratio is to increase the number of modes in the vertical direction. Due to the slow diffusion of heat for large aspect ratio enclosures, the onset of instability occurs much later in comparison to a model with smaller geometric dimensions and computation of asymptotic dynamics of the flow field becomes challenging for the prototype. We use dynamical similarity to show that similar events can be obtained with a model which facilitates the attainment of asymptotic states. The time history at specific points in the flow field shows that for Ra on the order of $10^{6}$ the temperature and flow fields oscillate periodically, however for Ra on the order of $10^{9}$ there is aperiodic behavior with intermittency in the flow field which is indicative of a transition to a chaotic state. Scaling of the temperature magnitude, for the parametric range of the model and prototype, indicates that there is a higher heat flux absorption on the boundaries than the nominal value of $34.9 \mathrm{erg} / \mathrm{cm}^{2}-\mathrm{s}$ or approximately $1 \mathrm{~mW}$ of background ambient heat flow. However, the parametic study shed light on the basic flow field dynamics associated with heat entrapment and can be used in future studies to address effect of increased heat flux at the boundaries. One of the highlights of the parametric study is the finding that transition to asymmetry occurs for Rayleigh numbers relevant to heat entrapment, this implies that axi-symmetric models are to be used with caution, since they are applicable to a very narrow parametric range near the critical Rayleigh number.

\section{References}

1. Bolshinskiy, L.G., Hastings, L.J., Statham, G., "Cryogenic Capillary Screen Heat Entrapment," Cryogenics, Vol. 48, 2008, pp. 232-237.

2. Tellep, D.M. and Harper, E.Y., "Approximate Analysis of Propellant Stratification," AIAA Journal, Vol. 1, No. 8, 1963, pp. 1954-1956.

3. Vliet, G.C., Brogan, T.J., Sheppard, T.S., Morse, F.H., and F.L. Hines, "Stratified Layer Flow Model - A Numerical Approach To Temperature Stratification In Liquids Contained In Heated Vessels," Aerospace Sciences Meeting, N.Y., N.Y. Jan. 20-22, Preprint No. 64-37, 1964.

4. Vliet, G.C., "Stratification with Bottom Heating," J. Spacecraft, Vol. 3, No.7, 1966, pp. 1142-1144.

5. Evans, L.B., Matulevicius, E.S., and Pan, P.C., "Reduction of Thermal Stratification by Nonuniform Wall Heating," AIChE Journal, Vol. 17, No. 3, 1971, pp. 752-753.

6. Evans, L.B., and Stefany, N.E., "An Experimental Study of Transient Heat Transfer To Liquids In Cylindrical Enclosures,” Chem. Eng. Prog. Symposium Ser. Vol. 62, No. 64, 1966, pp. 209-215.

7. Barakat, H.Z., Clark, J.A., "Analytical and Experimental Study of The Transient Laminar Natural Convection Flows in Partially Filled Liquid Containers," Proceedings of the Third International Heat Transfer Conference, Vol. II, Chicago, Illinois, Aug. 7-12, 1966, Papers 41-80.

8. Polyzhaev, V.I., "Nonstationary Laminar Heat Convection In A Closed Domain For a Given Heat Flux," Izvestiya Akademii Nauk SSSR, Meckanika Zhidkosti I Gaza, No. 4, 1970, pp. 109-117. 
9. Polyzhaev, V.I., "Convective Interaction In a Cylindrical Vessel Partially Filled With Liquid And With Heat Supplied To The Side Surface, Free Surface, And Bottom," Izvestiya Akademii Nauk SSSR, Meckanika Zhidkosti Gaza, No. 4, 1972, pp. 81-88.

10. Elder, J.W., "Laminar Free Convection In A Vertical Slot," J. Fluid Mech., Vol. 23, Part 1, 1965, pp. 77-98.

11. Elder, J.W., "Turbulent Free Convection In A Vertical Slot,” J. Fluid Mech., Vol. 23, Part 1, 1965, pp. 99-111.

12. Seki, N., Fukusako, S., Inaba, H., "Visual Observation of Natural Convection Flow In a Narrow Vertical Cavity,” J. Fluid Mech., Vol. 84, Part 4, 1978, pp. 695-704.

13. Akiyama, M., Hwang, G.J., Cheng, K.C., "Experiments On The Onset Of Longitudinal Vortices in Laminar Forced Convection Between Horizontal Plates,” J. Heat Transfer, Vol. 93, 1971, pp. 335341.

14. Chandrasekhar, S., Hydrodynamic and Hydromagnetic Stability, Oxford University Press, Oxford, UK, 1961, pp. 9-71.

15. Boris, J.P., and Book, D.L., "Flux-Corrected Transport I: SHASTA-A Fluid Transport Algorithm That Works," J. Comp. Phys., Vol. 11, Jan. 1973, pp. 139-174. 

